\title{
Bovine Colostrum Applications in Sick and Healthy People: A Systematic Review
}

\author{
Monica Guberti ${ }^{1,2}\left(\right.$, Stefano Botti ${ }^{3, *} \mathbb{0}$, Maria Teresa Capuzzo ${ }^{2}$, Sara Nardozi ${ }^{2}$, Andrea Fusco ${ }^{1}$, Andrea Cera ${ }^{4}$, \\ Laura Dugo $^{5}{ }^{-}$, Michela Piredda ${ }^{6}$ and Maria Grazia De Marinis ${ }^{6}$ \\ 1 Research and EBP Unit, Health Professions Department, Azienda USL-IRCCS di Reggio Emilia, \\ Via Amendola, 2-42122 Reggio Emilia, Italy; monica.guberti@ausl.re.it (M.G.); andrea.fusco@ausl.re.it (A.F.) \\ 2 Department of Biomedicine and Prevention, University of Rome Tor Vergata, Via di Montpellier, \\ 1-00133 Rome, Italy; mariateresa.capuzzo@libero.it (M.T.C.); saranardozi95@gmail.com (S.N.) \\ 3 Hematology Unit, Azienda USL-IRCCS di Reggio Emilia, Via Amendola, 2-42122 Reggio Emilia, Italy \\ 4 ASST-Azienda Socio-Sanitaria Territoriale di Mantova, Strada Lago Paiolo, 10-46100 Mantova, Italy; \\ andrea.cera@asst-mantova.it \\ 5 Unit of Food Science and Nutrition, Department of Science and Technology for Humans and the Environment, \\ University Campus Bio-Medico of Rome, Via Alvaro del Portillo, 21-00128 Rome, Italy; 1.dugo@unicampus.it \\ 6 Research Unit Nursing Science, University Campus Bio-Medico of Rome, Via Alvaro del Portillo, \\ 21-00128 Rome, Italy; m.piredda@unicampus.it (M.P.); m.demarinis@unicampus.it (M.G.D.M.) \\ * Correspondence: stefano.botti@ausl.re.it; Tel.: +39-05-2229-6661; Fax: +39-05-2229-5655
}

Citation: Guberti, M.; Botti, S.; Capuzzo, M.T.; Nardozi, S.; Fusco, A.; Cera, A.; Dugo, L.; Piredda, M.; De Marinis, M.G. Bovine Colostrum Applications in Sick and Healthy People: A Systematic Review. Nutrients 2021, 13, 2194. https:// doi.org/10.3390/nu13072194

Academic Editor: Raymond Playford

Received: 26 May 2021

Accepted: 22 June 2021

Published: 25 June 2021

Publisher's Note: MDPI stays neutral with regard to jurisdictional claims in published maps and institutional affiliations.

Copyright: (c) 2021 by the authors. Licensee MDPI, Basel, Switzerland. This article is an open access article distributed under the terms and conditions of the Creative Commons Attribution (CC BY) license (https:// creativecommons.org/licenses/by/ $4.0 /)$.

\begin{abstract}
Colostrum is the first secretion of mammalian glands during the early period after birth giving. Its components are biologically active and have beneficial effects on new-born growth and well-being. Bovine colostrum has the highest concentration of these substances and its supplementation or application may provide health benefits. This systematic review was conducted to update current knowledge on bovine colostrum effects including all administration routes on healthy and sick subjects. Full texts or abstracts of twenty-eight papers as reports of systematic reviews, randomized controlled trials, observational studies and case series were included after searches in Medline, Embase, Cochrane Library and Cinahl databases. The full texts of selected studies were assessed for quality using validated tools and their results were summarized in different categories. Studies were highly heterogeneous as regards to population, intervention, outcome and risk of bias. Bovine colostrum topical application was shown effective on vaginal dryness related symptoms limitation. Its use as food supplement showed interesting effects preventing upper respiratory illness in sportsmen, modulating immune system response and reducing intestinal permeability in healthy and sick subjects. Conflicting results were provided in pediatric population and little evidence is available on its use with older adults. Further studies are mandatory to better understand all factors influencing its activity.
\end{abstract}

Keywords: bovine colostrum; whey; milk; food supplementation; health improvement; physical performance; immune system; systematic review

\section{Introduction}

Colostrum is the secretion produced by the mammary gland immediately following parturition; it provides to infants sustenance, enhances their protection against pathogens, ensures immune system development, and provides to growth, maturation, and repair of several tissues [1]. The composition and physical properties of colostrum are highly variable due to a number of factors, including individuality, breed, parity, pre-partum nutrition, length of the dry period of cows and time post-partum [2-6]. It differs from milk as it contains less lactose and more fat, protein, peptides, non-protein nitrogen, ash, vitamins and minerals, hormones, growth factors, cytokines and nucleotides. The composition of both human and Bovine Colostrum (BC) have been largely studied highlighting higher concentrations than mature milk of a wide variety of biologically active substances [7]. 
These retain their activity while passing the gastrointestinal tract, carrying out beneficial effect on the intestinal functions [8-10] mainly attributable to immune-modulatory [11,12], antimicrobial $[8,13]$ and anti-inflammatory [12] activities. Immunoglobulin antibodies are the main immune components of the acquired immune system present in colostrum and milk. Colostrum is characterized by its very high concentration of immunoglobulin $G$ (IgG), which is of particular importance as it confers passive immunity to the neonate immediately following parturition $[6,8,14,15]$. The most abundant immunoglobulin class in bovine milk and colostrum is IgG1 [16]. In contrast, IgA and IgM are present at much reduced concentrations in bovine colostrum and milk [17]. Lactoferrin and lactoperoxidase have significant antimicrobial effects [18] and other components such as cytokines and interleukins are involved in inflammatory regulation processes and contribute to infection control also [12]. The highly glycosylated polypeptide secretory component [19] is another immune factor present in colostrum and milk that interacts with the adaptive immune system. Secretory component derived from a portion of the IgA receptor not only enhances IgA functionality when it is attached to IgA [20] but may have direct protective properties itself. Lysozyme is a lytic enzyme that plays a role in the innate immune system by attacking peptidoglycan cell constituents found primarily in gram-positive bacteria, leading to bacterial lysis $[18,21]$. Bovine colostrum has shown an emerging role as a food supplement due to its healing properties targeted to boost the immune systems in both healthy and chronically ill patients $[1,18,22]$. Colostrum has been known for centuries for its health benefits [23]. Literature also showed that the active components in BC were 100 to 1000-fold more concentrated than in human one. This means that even human infants can rely on cow or buffalo colostrum to gain health benefits [22,24]. Topical application of the colostrum constituents has proven promising for open wound healing [25]. It has been suggested that nucleotides, epidermal growth factor (EGF), transforming growth factor (TGF) and insulin like growth factor-1 (IGF-1) promote cellular and skin growth and also help in repairing DNA and RNA damage [6]. A patent has also been granted for the use of a colostrum fraction to accelerate wound healing [26]. For all these reasons, BC has found its application in the prevention and treatment of several inflammatory diseases, such as those affecting the gastrointestinal and respiratory systems of adults and children [27]. In addition, bovine colostrum derivatives have been used for the treatment of rheumatoid arthritis. Studies have shown benefits of BC supplementation in healthy populations such as children, adolescents [13] and sporting individuals, more precisely because it can boost their immune, digestive, and hormonal systems, as well as it may improve their physical performances [11]. The aim of this literature review is to assess the evidence supporting the bovine colostrum administration in clinical and not clinical settings in order to provide additional knowledge on its use and updating results of the last review on this topic [21].

\section{Materials and Methods}

\subsection{Search Strategy}

This review updated the last one published in 2014 [21]. The PICO method ( $\mathrm{P}=\mathrm{Pop}-$ ulation, $\mathrm{I}=$ Intervention, $\mathrm{C}=\mathrm{Control}, \mathrm{O}=$ Outcome) was used to make the research question. Healthy and not-healthy subjects were included in the Population frame; Interventions were administration of $\mathrm{BC}$ (any formulation) with the aim to improve physical performances or health; all Control formulae including placebo and no-treatment were considered while the Outcomes were undefined. A literature search was conducted within the following databases: Medline, Embase, Cochrane Library and Cinahl. The following search strategy was used in Medline and adapted to the other databases: ("Colostrum" [Mesh] OR colostrum OR colostrums) AND (bovine OR cow OR cows OR cattle), records were filtered by time frame (from 1 January 2013 to 31 December 2020), language (English) and involved subjects (Human). Reference lists of included papers were manually checked in order to find further records. Full texts papers were considered in the analysis while abstracts were discussed separately. 


\subsection{Inclusion and Exclusion Criteria}

Any population (adult, pediatric, aged, sick and healthy subjects), administration route (enteral, topical, etc.), endpoints and outcomes were considered to find results on BC use. Systematic reviews (SR), randomized controlled trials (RCTs), other clinical trials (CTs), observational studies (OS) and case series (CSs) were included. Full text papers or abstracts were considered as well as studies, investigating BC effects either as dietary supplement or as topical application. Records concerning hyper-immune colostrum, which are produced by administering polyvalent bacterial vaccines to cows with the aim of stimulating the production of higher levels of total and antigen-specific immunoglobulins [28] were excluded as well as those exploring the effects of composite products where colostrum was mixed with other bioactive substances. Any type of change, measurable in terms of health condition or physical performance, was considered as an eligible outcome for this review. The PRISMA Statement [29] was followed for results selection and reporting.

\subsection{Study Selection and Quality Assessment}

Study selection was performed by removing duplicates and non-relevant records, or protocols, first, on title and abstract basis and, second, after full-text reading (Figure 1). The quality assessment of the studies included was conducted with the aid of validated tools. The Effective Public Health Practice Project (EPHPP) [30] was used for clinical trials, randomized clinical trials (RCTs) and observational studies, while A Measurement Tool to Assess Systematic Reviews-2 (AMSTAR-2) [31] was used in order to assess reviews. The EPHPP is widely used in evaluating RCTs and clinical trials, with an excellent degree of inter-rating reliability [30]. This tool consists of six domains (selection bias, study design, confounders, blinding, data collection methods, withdrawals and drop-outs), whose sum of scores constitutes the overall quality rating, where (1) "strong" includes papers without weak subscale grading, (2) "moderate" includes those with at least one weak grading, and (3) "weak" includes two or more weak rating of the sub-scales. The AMSTAR-2 tool consists of 16 items with an overall rating based on weaknesses in critical domains. The overall rating is classified as "high" (No or one non-critical weakness), "moderate" (More than one non-critical weakness) "low" (One critical flaw with or without non-critical weaknesses) and "critically low" (More than one critical flaw with or without non-critical weaknesses). Quality assessment of the included papers with EPHPP and AMSTAR-2 was performed by two independent reviewers, who met to discuss and solve any discrepancies in the study evaluation or results interpretation. With the aim to intercept minimal differences in studies quality and to better define level of evidence, two additional tools were applied. The risk of bias of the included RCTs was assessed by two different independent reviewers using the Cochrane Collaboration Risk of Bias Tool (CCRBT) including the following seven domains: random sequence generation; allocation concealment; blinding of participants and personnel; blinding of outcome assessment; incomplete outcome data; selective reporting and other biases. The CCRBT is different from EPHPP as, for example, outcome reporting is evaluated more in detail than in the EPHPP, which gives no specific evaluation on absence from reporting bias [30]. Then, the level of evidence was assessed by two independent reviewers using the 2011 Oxford Centre for Evidence Based Medicine level of evidence method (OCEBM), which was developed by an international group and took into account feedback from clinicians, patients, and researchers. It allows to rapidly find the likely best evidence encouraging clinicians, researchers and patients to autonomously assess evidence [32] (Table 1). 


\section{Identification of studies via databases and registers}

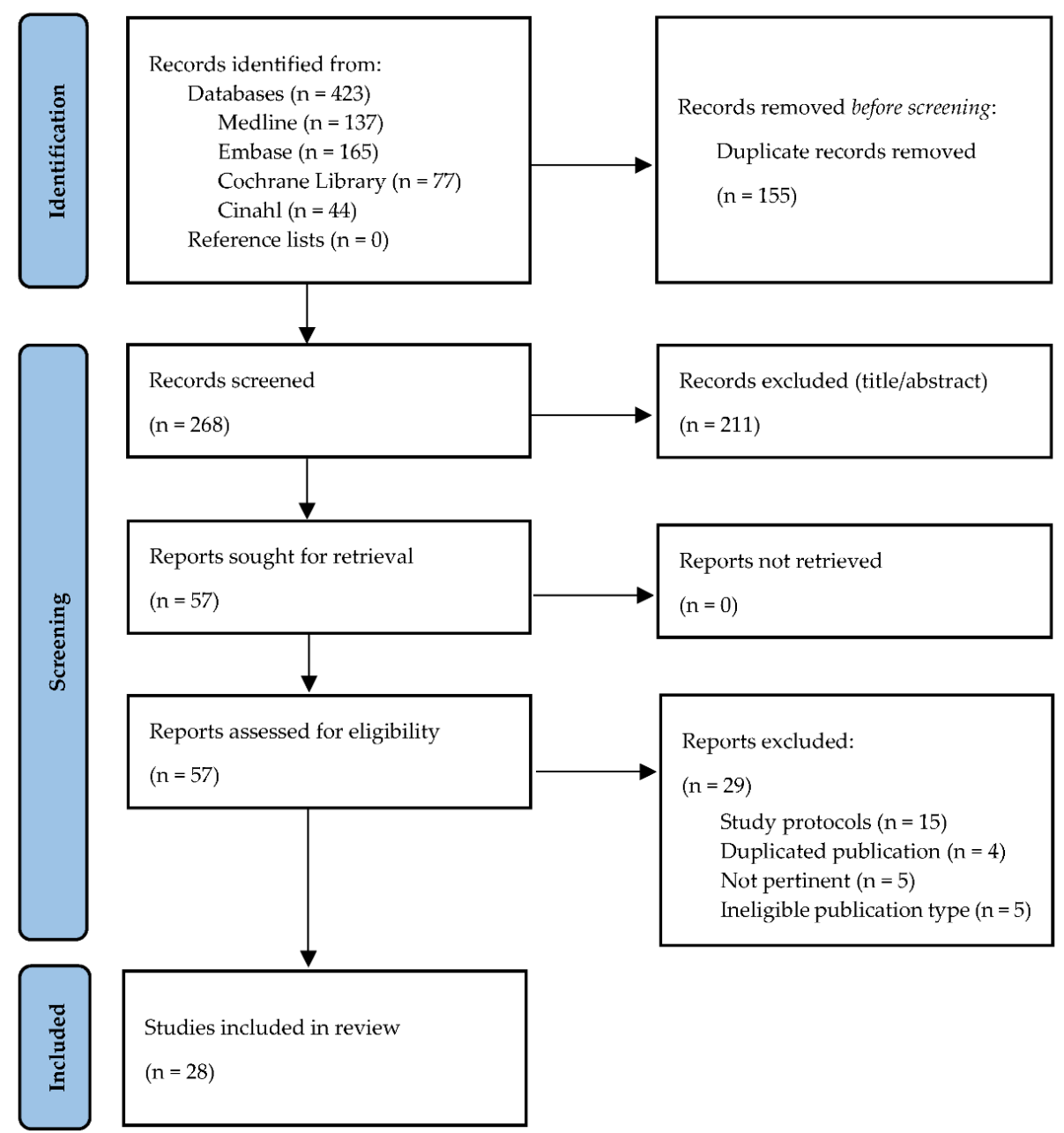

Figure 1. PRISMA flowchart of the study selection process.

Table 1. OCEBM Level of Evidence 2011 [32].

\section{Level 1 *}

Systematic review

of randomized trials or n-of- 1 trials

Level 2 *

Evidence Level (Treatment Benefits)

Randomized trial or observational study with dramatic effect

Level 3 *

Non-randomized controlled cohort/follow-up study **

\section{Level 4 *}

Case-series, case control studies, or historically controlled studies **

Level 5 *

Mechanism-based reasoning

* Level may be graded down on the basis of study quality, imprecision, indirectness (study PICO does not match questions PICO), because of inconsistency between studies, or because the absolute effect size is very small; Level may be graded up if there is a large or very large effect size. ${ }^{* *}$ As always, a systematic review is generally better than an individual study. 


\section{Results}

\subsection{Paper Selection and Categorization}

The literature searching identified 423 records: 137 from Medline, 165 from Embase, 77 from the Cochrane Library and 44 from Cinahl. After removal of 155 duplicates, 268 articles were considered appropriate in accordance to the screening of title, abstract and full-text reading. Finally, 28 articles were included in the review. Figure 1 represents the Flow Chart of the study selection process. Twenty-four out the 28 studies included the full texts [33-55], while 4 were available as abstracts only [56-59]. The majority of these articles involved participants from Europe $(n=14 ; 52 \%), 6(22 \%)$ from Asia, $3(11 \%)$ from Africa $1(4 \%)$ from America and $3(11 \%)$ from Oceania. Twenty-four studies involving 1057 participants were RCTs (4 available as abstracts only), 3 were observational studies with 588 patients and 1 was a systematic review. Therefore, the papers were classified by publication type (full texts or abstracts), BC administration route (systemic oral or topical) and study population. BC topical application in uro-gynecology [33-35] (Table 2), BC as dietary supplement in the sporting population [36-46] (Table 3), BC as dietary supplement in the pediatrics and preterm infants [47-53] (Table 4), BC as dietary supplement in the elderly [54] (Table 5), BC administration in critically ill patients [55] (Table 6), abstracts [56-59] (Table 7) and then a systematic review on BC clinical applications [21] (Table 8). In Table 9 the risk of bias of the included (full texts) RCTs was reported according to the CCRBT method.

\subsection{Studies Heterogeneity}

Three studies (1 RCT, 2 OS) were on topical applications of BC-based creams on various vaginal conditions and recruiting only female participants with a mean age ranged from 27 to 61 years [33-35]. Twenty-four papers (23 RCTs, 1 OS) assessed the efficacy of BC as dietary supplement in various populations [36-59], including diseased ones [47-53,55-59] and healthy people [36-46,54]. Of these papers, 1 included 3 RCTs (please, note that it is considered as one study in this review) [39], 11 recruited only sporting males with a mean age ranged from 21 to 51 years [36-46], 11 involved pediatric subjects affected by various clinical conditions [47-53,56-59], 1 was on critically ill patients and 1 on healthy elderlies. The participants involved in the 23 full texts of primary studies included received 11 different commercially available $\mathrm{BC}$ formulas, while one study experimented the effects of "fresh" BC [47]. In addition, BC was administered at different dosages ranging from 1 to $60 \mathrm{~g} /$ day in adult setting (13 RCTs) [36-46,54,55] and from $0.014 \mathrm{~g} /$ day to $4.5 \mathrm{~g} / \mathrm{kg} /$ day in the 7 pediatric ones ( 7 studies) [47-53]. Colostum and placebos were administered majorly as beverages supplementing meals in clinical settings (12 studies) [47-53,55-59] and supporting physical performances or specific training programs in healthy people (12 RCTs). Refs. [36-46,54] The control groups of the 19 included full texts RCTs received 9 different placebos including isoenergetic/isomacronutrient formulas, whey or mixed milk matrix, whey protein concentrated formulas, corn flour, maltodextrin. Limited information on BC and placebo products were available by the 4 RCTs (all involving pediatric subjects) available as abstracts [56-59]. Surrogate outcomes were used in 15 studies $[37-47,50,51,54,55]$ while $8[33-36,48,49,52,53]$ assessed morbidity outcomes. The 4 abstracts measured primary outcomes. Refs. [56-59] As explained above, the included papers were very heterogeneous precluding any meta-analysis in this review. 
Table 2. BC topical applications in uro-gynecology setting.

\begin{tabular}{|c|c|c|c|c|c|c|c|c|c|c|c|}
\hline Authors & Study Design & $\begin{array}{l}\text { Population } \\
\text { Number } \\
\text { Groups } \\
\text { Gender } \\
\text { Mean Age }\end{array}$ & $\begin{array}{l}\text { Intervention } \\
\text { Matrix }\end{array}$ & $\begin{array}{l}\text { TG Size } \\
\text { Dosage } \\
\text { Frequency } \\
\text { Duration }\end{array}$ & $\begin{array}{l}\text { Control } \\
\text { Matrix }\end{array}$ & $\begin{array}{l}\text { CG Size } \\
\text { Dosage } \\
\text { Frequency } \\
\text { Duration }\end{array}$ & $\begin{array}{c}\text { Endpoints } \\
\text { Data Collection } \\
\text { Tools }\end{array}$ & $\begin{array}{l}\text { Adverse } \\
\text { Events }\end{array}$ & Results & OCEBM & ЕРНPP \\
\hline $\begin{array}{l}\text { Nappi, R. E., } \\
\text { et al. (2016) } \\
\text { [33] }\end{array}$ & $\mathrm{RCT}$ & $\begin{array}{l}\text { Women with } \\
\text { vaginal } \\
\text { dryness. } \\
n=95 ;>18 \text { ys }\end{array}$ & $\begin{array}{c}\text { Monurelle } \\
\text { Biogel }^{\circledR}\end{array}$ & $\begin{array}{c}\mathrm{TG}(n=48) \\
5 \mathrm{~mL}, 1-2 \text { time } \\
\text { per day during } \\
\text { intermenstrual } \\
\text { period } \\
\text { (23 days) }\end{array}$ & $\begin{array}{l}\text { No-treatment, } \\
\text { nonactive } \\
\text { lubricants on } \\
\text { demand were } \\
\text { allowed }\end{array}$ & CG $(n=47)$ & $\begin{array}{c}\text { PE: Vaginal } \\
\text { discomfort (VRS) } \\
\text { SE: (1) Symptoms } \\
\text { (VRS); } \\
\text { (2) Vaginal health } \\
\text { (VHI mean } \\
\text { sum score); } \\
\text { (3) Sexual function } \\
\text { (FSFI); } \\
\text { (4) Sexual distress } \\
\text { (FSDS-R). }\end{array}$ & $\begin{array}{l}\text { No severe or } \\
\text { serious AEs } \\
\text { Mild AEs } \\
\text { in } 16.7 \% \\
\text { CG: AEs } \\
\text { in } 8.5 \%\end{array}$ & $\begin{array}{c}\downarrow \text { Vaginal } \\
\text { discomfort } \\
\downarrow \text { Vaginal } \\
\text { symptoms } \\
\uparrow \text { Vaginal health } \\
\text { Sexual function } \\
\text { improved } \\
\downarrow \text { Sexual distress }\end{array}$ & Level 1 & 1 \\
\hline $\begin{array}{l}\text { Schiavi, M. C., } \\
\text { et al. (2019) } \\
\text { [34] }\end{array}$ & Retrospective & $\begin{array}{l}\text { Postmenopausal } \\
\text { women with } \\
\text { VVA. } n=172 \\
\text { mean age } \\
60.8 \text { ys }\end{array}$ & $\begin{array}{c}\text { Monurelle } \\
\text { Biogel }^{\circledR}\end{array}$ & $\begin{array}{l}5 \mathrm{~mL} \text { once } \\
\text { daily for } \\
12 \text { weeks }\end{array}$ & No CG & No CG & $\begin{array}{l}\text { PE: Vaginal health } \\
\text { (VHI); } \\
\text { SE: (1) Sexual } \\
\text { function (FSFI); (2) } \\
\text { Sexual distress } \\
\text { (FSDS); (3) Urinary } \\
\text { symptoms } \\
\text { (4) Urogenital } \\
\text { distress (UDI-6); } \\
\text { (5) Overactive } \\
\text { bladder symptoms } \\
\text { (OAB-Q) } \\
\text { (6) QoL (HRQL) }\end{array}$ & $\begin{array}{c}\text { No significant } \\
\text { AEs }\end{array}$ & $\begin{array}{c}\uparrow \text { Vaginal health } \\
\text { Sexual function } \\
\text { improved } \\
\downarrow \text { Sexual distress } \\
\downarrow \text { Urinary } \\
\text { symptoms } \\
\downarrow \text { Urogenital } \\
\quad \text { distress } \\
\downarrow \text { OAB symptoms } \\
\uparrow \text { QoL }\end{array}$ & Level 3 & 3 \\
\hline $\begin{array}{l}\text { Stefani, C., } \\
\text { et al. (2014) } \\
\text { [35] }\end{array}$ & Retrospective & $\begin{array}{c}\text { Women } \\
\text { diagnosed as } \\
\text { CIN1. } \\
n=256 ; \text { mean } \\
\text { age } 37.7 \text { ys }\end{array}$ & $\begin{array}{c}\text { Ginedie }^{\circledR} \\
\text { vaginal tablets }\end{array}$ & $\begin{array}{l}\text { twice/week at } \\
\text { bedtime for } \\
6 \text { months. }\end{array}$ & No CG & No CG & $\begin{array}{l}\text { ORR to negative } \\
\text { histology (Cervical } \\
\text { cytology) }\end{array}$ & NR & $75.5 \%$ ORR & Level 4 & 3 \\
\hline
\end{tabular}

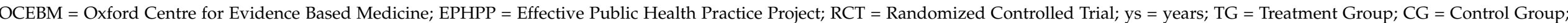

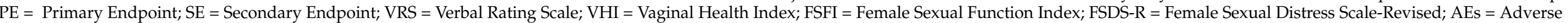

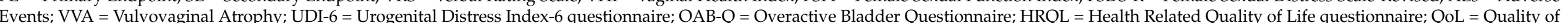
Life; CIN1 = Atypical squamous intraepithelial lesions; NR = Not Reported; ORR = Overall Reduction Rate. Symbols: $\uparrow=$ increased; $\downarrow=$ decreased 
Table 3. BC as dietary supplement in the sporting population.

\begin{tabular}{|c|c|c|c|c|c|c|c|c|c|c|c|}
\hline Authors & Study Design & $\begin{array}{c}\text { Population } \\
\text { Number } \\
\text { Groups } \\
\text { Gender } \\
\text { Mean Age }\end{array}$ & $\begin{array}{l}\text { Intervention } \\
\text { Matrix }\end{array}$ & $\begin{array}{l}\text { TG size } \\
\text { Dosage } \\
\text { Frequency } \\
\text { Duration }\end{array}$ & $\begin{array}{l}\text { Control } \\
\text { Matrix }\end{array}$ & $\begin{array}{l}\text { CG Size } \\
\text { Dosage } \\
\text { Frequency } \\
\text { Duration }\end{array}$ & $\begin{array}{l}\text { Endpoints/Data } \\
\text { Collection Tools }\end{array}$ & $\begin{array}{c}\text { Adverse } \\
\text { Events }\end{array}$ & Results & OCEBM & EPHPP \\
\hline $\begin{array}{l}\text { Jones, A. W., } \\
\text { et al. (2014) } \\
\text { [36] }\end{array}$ & RCT, DB, PC & $\begin{array}{c}\text { Regularly } \\
\text { exercising } \\
\text { males } \\
n=53 \text {; mean } \\
\text { age } 50.5 \text { ys } \\
\text { Colostrum } \\
(n=25) \text { vs. } \\
\text { Placebo } \\
(n=28)\end{array}$ & $\begin{array}{l}\text { BC (Neovite } \\
\text { UK, London) }\end{array}$ & $\begin{array}{l}\text { TG }(n=25) \\
20 \text { g/day } \\
12 \text { weeks. }\end{array}$ & $\begin{array}{l}\text { Isoenergetic/ } \\
\text { Isomacronutrient } \\
\text { placebo }\end{array}$ & $\begin{array}{c}\text { CG }(n=28) \\
20 \text { g/day } \\
12 \text { weeks. }\end{array}$ & $\begin{array}{l}\text { PE: (1) Incidence of } \\
\text { URI; } \\
\text { SE: (1) URI days; } \\
\text { (2) URI duration } \\
\text { (episodes) } \\
\text { (3) Immune- } \\
\text { system parameters } \\
\text { (4) Salivary } \\
\text { antimicrobial } \\
\text { proprieties } \\
\text { (sIgA/AMPs) } \\
\text { (5) Salivary } \\
\text { microbiome } \\
\text { composition }\end{array}$ & NR & $\begin{array}{c}\downarrow \text { URI incidence; } \\
\downarrow \text { URI days; } \\
\downarrow \text { salivary } \\
\text { bacterial load; } \\
\text { No significant } \\
\text { effects on: } \\
\text { severity and } \\
\text { duration of URI } \\
\text { episodes, } \\
\text { immune-system } \\
\text { parameters, } \\
\text { salivary } \\
\text { sIgA/AMPs }\end{array}$ & Level 2 & 1 \\
\hline \multirow{3}{*}{$\begin{array}{l}\text { Davison G., } \\
\text { et al. (2019) } \\
\text { [39] }\end{array}$} & $\begin{array}{c}\text { RCT, DB, PC, } \\
\text { CB, CO }\end{array}$ & $\begin{array}{c}\text { Recreationally } \\
\text { active males } \\
n=16 ; \text { mean } \\
\text { age } 25.0 \text { ys } \\
\text { CO after a } \\
\text { week }\end{array}$ & $\begin{array}{l}\text { BC (Neovite } \\
\text { UK, London) } \\
\text { during } 4.5 \mathrm{~h} \\
\text { long moderate } \\
\text { exercise }\end{array}$ & $\begin{array}{l}\text { TG ( } n=16): \\
\quad 40 \mathrm{~g}\end{array}$ & $\begin{array}{l}\text { Isoenergetic/ } \\
\text { Isomacronutrient } \\
\text { placebo } \\
\text { during } 4.5 \mathrm{~h} \\
\text { long moderate } \\
\text { exercise }\end{array}$ & $\begin{array}{l}\text { CG }(n=16) \\
\quad 40 \mathrm{~g}\end{array}$ & IGF-1 blood levels & NR & $\begin{array}{l}\text { No significant } \\
\text { effects on IGF-1 } \\
\text { blood levels }\end{array}$ & Level 2 & \multirow{3}{*}{1} \\
\hline & RCT, DB, PC & $\begin{array}{c}\text { Recreationally } \\
\text { active males } \\
n=20 ; \text { mean } \\
\text { age } 28.0 \text { ys }\end{array}$ & $\begin{array}{c}\text { BC (Neovite }{ }^{\circledR} \\
\text { UK, London) + } \\
\text { training } \\
\text { program }\end{array}$ & $\begin{array}{l}\text { TG }(n=10) \\
20 \text { g/day } \\
4 \text { weeks }\end{array}$ & $\begin{array}{l}\text { Isoenergetic/ } \\
\text { Isomacronutrient } \\
\text { Placebo }+ \\
\text { training } \\
\text { program }\end{array}$ & $\begin{array}{c}\text { CG }(n=10) \\
20 \text { g/day } \\
4 \text { weeks }\end{array}$ & IGF-1 blood levels & NR & $\begin{array}{l}\text { No significant } \\
\text { effects on IGF-1 } \\
\text { blood levels }\end{array}$ & Level 3 & \\
\hline & RCT, DB, PC & $\begin{array}{c}\text { Recreationally } \\
\text { active males } \\
n=57 ; \text { mean } \\
\text { age NR } \\
\text { Colostrum } \\
\text { ( } n=\mathrm{NR}) \text { vs. } \\
\text { Placebo } \\
\text { ( } n=\mathrm{NR}) \\
n=4 \text { excluded } \\
\text { from the } \\
\text { analysis }\end{array}$ & $\begin{array}{c}\text { BC (Neovite }{ }^{\circledR} \\
\text { UK, London) + } \\
\text { training } \\
\text { program }\end{array}$ & $\begin{array}{c}\text { TG }(n=25): \\
\text { BC } \\
20 \text { g/day } \\
12 \text { weeks }\end{array}$ & $\begin{array}{c}\text { Isoenergetic/ } \\
\text { Isomacronutrient } \\
\text { Placebo + } \\
\text { training } \\
\text { program }\end{array}$ & $\begin{array}{c}\text { CG }(n=28) \\
20 \text { g/day } \\
12 \text { weeks }\end{array}$ & IGF-1 blood levels & NR & $\begin{array}{l}\text { No significant } \\
\text { effects on IGF-1 } \\
\text { blood levels }\end{array}$ & Level 3 & \\
\hline
\end{tabular}


Table 3. Cont

\begin{tabular}{|c|c|c|c|c|c|c|c|c|c|c|c|}
\hline Authors & Study Design & $\begin{array}{c}\text { Population } \\
\text { Number } \\
\text { Groups } \\
\text { Gender } \\
\text { Mean Age }\end{array}$ & $\begin{array}{l}\text { Intervention } \\
\text { Matrix }\end{array}$ & $\begin{array}{l}\text { TG size } \\
\text { Dosage } \\
\text { Frequency } \\
\text { Duration }\end{array}$ & $\begin{array}{l}\text { Control } \\
\text { Matrix }\end{array}$ & $\begin{array}{l}\text { CG Size } \\
\text { Dosage } \\
\text { Frequency } \\
\text { Duration }\end{array}$ & $\begin{array}{l}\text { Endpoints/Data } \\
\text { Collection Tools }\end{array}$ & $\begin{array}{l}\text { Adverse } \\
\text { Events }\end{array}$ & Results & OCEBM & EPHPP \\
\hline $\begin{array}{l}\text { Halasa, M., } \\
\text { et al. (2017) } \\
\text { [40] }\end{array}$ & RCT, DB, PC & $\begin{array}{c}\text { Competitive } \\
\text { athletic males } \\
n=16 ; \text { mean } \\
\text { age } 27.5 \text { ys }\end{array}$ & $\begin{array}{c}\text { Freeze-dried } \\
\text { whole BC } \\
\text { obtained } \\
\text { within } 2 \mathrm{~h} \text { of } \\
\text { calf delivery } \\
\text { (Genactiv } \\
\text { Poznan, } \\
\text { Poland) was } \\
\text { packaged in } \\
\text { pouches BC } \\
500 \mathrm{mg} \text { and } \\
\text { desiccated } \\
\text { banana } \\
500 \mathrm{mg} .\end{array}$ & $\begin{array}{l}\text { TG }(n=8) \\
1 \text { g/day } \\
20 \text { days }\end{array}$ & $\begin{array}{l}\text { Identical } \\
\text { pouches } \\
\text { (500 mg of } \\
\text { dehydrated } \\
\text { whey } \\
\text { and } 500 \mathrm{mg} \text { of } \\
\text { desiccated } \\
\text { banana) were } \\
\text { used as the } \\
\text { placebo. }\end{array}$ & $\begin{array}{l}\text { CG }(n=8) \\
1 \text { g/day } \\
20 \text { days }\end{array}$ & $\begin{array}{l}\text { Gut permeability: } \\
\text { sugar absorption } \\
\text { test, zonulin } \\
\text { concentration }\end{array}$ & $\begin{array}{l}\text { No AEs in the } \\
\text { TG. Mild AEs } \\
\text { in } 50 \% \text { of CG }\end{array}$ & $\begin{array}{c}\downarrow \text { sugar } \\
\text { absorption } \\
\downarrow \text { zonulin } \\
\text { concentration }\end{array}$ & Level 3 & 1 \\
\hline $\begin{array}{c}\text { Jones, A. W., } \\
\text { et al. (2019) } \\
\text { [38] }\end{array}$ & RCT, DB, PC & $\begin{array}{c}\text { Recreationally } \\
\text { active males } \\
n=34 ; \text { mean } \\
\text { age NR } \\
\text { Colostum } \\
(n=17) \text { vs. } \\
\text { Placebo } \\
(n=17) \\
n=3 \text { excluded } \\
\text { from the } \\
\text { analysis }\end{array}$ & $\begin{array}{l}\mathrm{BC}+\text { water }+ \\
\text { training } \\
\text { program } \\
\text { Day 28: } 2 \mathrm{~h} \text { of } \\
60 \% \text { maximal } \\
\text { aerobic } \\
\text { capacity and } \\
\text { immune } \\
\text { system } \\
\text { sensitisation } \\
\text { Day 56: } \\
\text { elicitation of } \\
\text { immunity }\end{array}$ & $\begin{array}{l}\text { TG }(n=15) \\
20 \mathrm{~g} / \text { day } \\
58 \text { days. }\end{array}$ & $\begin{array}{l}\text { isoenergetic/ } \\
\text { isomacronutrient } \\
\text { Placebo + } \\
\text { training } \\
\text { program } \\
\text { Day 28: } 2 \mathrm{~h} \text { of } \\
60 \% \text { maximal } \\
\text { aerobic } \\
\text { capacity and } \\
\text { immune } \\
\text { system } \\
\text { sensitisation } \\
\text { Day } 56: \\
\text { elicitation of } \\
\text { immunity }\end{array}$ & $\begin{array}{c}\text { CG }(n=16) \\
20 \mathrm{~g} / \text { day } \\
58 \text { days }\end{array}$ & $\begin{array}{l}\text { PE: Cell mediated } \\
\text { response following } \\
\text { prolonged exercise } \\
\text { (skinfold } \\
\text { reactivity) } \\
\text { SE: (1) IGF-1 blood } \\
\text { levels } \\
\text { (2) Immune cell } \\
\text { counts } \\
\text { (3) Biochemical } \\
\text { parameters }\end{array}$ & NR & $\begin{array}{c}\text { limmune } \\
\text { sensitivity } \\
\text { decreasing after } \\
\text { prolonged } \\
\text { exercise } \\
\text { No significant } \\
\text { effects on in-vivo } \\
\text { immune } \\
\text { responsiveness, } \\
\text { IGF-1 blood } \\
\text { levels, Immune } \\
\text { cell counts and } \\
\text { other } \\
\text { biochemical } \\
\text { parameters }\end{array}$ & Level 2 & 1 \\
\hline
\end{tabular}


Table 3. Cont

\begin{tabular}{|c|c|c|c|c|c|c|c|c|c|c|c|}
\hline Authors & Study Design & $\begin{array}{c}\text { Population } \\
\text { Number } \\
\text { Groups } \\
\text { Gender } \\
\text { Mean Age }\end{array}$ & $\begin{array}{l}\text { Intervention } \\
\text { Matrix }\end{array}$ & $\begin{array}{l}\text { TG size } \\
\text { Dosage } \\
\text { Frequency } \\
\text { Duration }\end{array}$ & $\begin{array}{l}\text { Control } \\
\text { Matrix }\end{array}$ & $\begin{array}{l}\text { CG Size } \\
\text { Dosage } \\
\text { Frequency } \\
\text { Duration }\end{array}$ & $\begin{array}{l}\text { Endpoints/Data } \\
\text { Collection Tools }\end{array}$ & $\begin{array}{l}\text { Adverse } \\
\text { Events }\end{array}$ & Results & OCEBM & EPHPP \\
\hline $\begin{array}{c}\text { Jones, A. W., } \\
\text { et al. (2015) } \\
\text { [37] }\end{array}$ & $\mathrm{RCT}, \mathrm{DB}, \mathrm{PC}$ & $\begin{array}{l}\text { Recreationally } \\
\text { active males } \\
n=20, \text { mean } \\
\text { age } 28.0 \text { ys }\end{array}$ & $\begin{array}{l}\text { BC (Neovite } \\
\text { UK, London) }\end{array}$ & $\begin{array}{c}\text { TG }(n=10) \\
20 \mathrm{~g} / \text { day } \\
4 \text { weeks }\end{array}$ & $\begin{array}{l}\text { isoenergetic/ } \\
\text { isomacronutrient } \\
\text { Placebo }\end{array}$ & $\begin{array}{c}\text { CG }(n=10) \\
20 \mathrm{~g} / \text { day } \\
4 \text { weeks }\end{array}$ & $\begin{array}{l}\text { PE: (1) In-vitro } \\
\text { blood neutrophil } \\
\text { function: fMLP } \\
\text { and PMA } \\
\text { (2) Mucosal } \\
\text { responses: sIgA } \\
\text { and AMP } \\
\text { SE: (1) Circulating } \\
\text { cells count } \\
\text { (2) Biochemical } \\
\text { parameters }\end{array}$ & NR & $\begin{array}{c}\text { Beneficial } \\
\text { in vitro effects } \\
\text { on receptor- } \\
\text { dependent } \\
\text { (fMLP- } \\
\text { stimulated) } \\
\text { oxidative burst } \\
\text { responses. } \\
\text { No in vitro effect } \\
\text { on PMA- } \\
\text { stimulated } \\
\text { oxidative burst, } \\
\text { sIgA and AMP. } \\
\text { No effects on } \\
\text { leukocyte } \\
\text { trafficking and } \\
\text { other } \\
\text { biochemical } \\
\text { parameters }\end{array}$ & Level 2 & 1 \\
\hline $\begin{array}{l}\text { Kotsis, Y., et al. } \\
\text { (2019) [42] }\end{array}$ & RCT, DB, PC & $\begin{array}{l}\text { Soccer players } \\
n=22 ; \text { mean } \\
\text { age } 21.1 \text { ys }\end{array}$ & $\begin{array}{c}\text { Commercial } \\
\text { BC } \\
378 \mathrm{Kcal}, 67 \mathrm{~g} \\
\text { protein, } 17 \mathrm{~g} \\
\text { carbohydrates } \\
\text { and } 4.7 \mathrm{~g} \mathrm{fat} \\
\text { per } 100 \mathrm{~g} \\
\text { Pre and post } \\
\text { supplementa- } \\
\text { tion LIST } \\
\text { exercise } \\
\text { program }\end{array}$ & $\begin{array}{c}\text { TG }(n=11) \\
3.2 \mathrm{~g} / \text { day } \\
6 \text { weeks. }\end{array}$ & $\begin{array}{l}\text { Commercial } \\
\text { whey protein } \\
369 \text { Kcal, } 90 \mathrm{~g} \\
\text { protein, } 1 \mathrm{~g} \\
\text { carbohydrates } \\
\text { and } 0.5 \mathrm{~g} \text { fat } \\
\text { per } 100 \mathrm{~g} \\
\text { Pre and post } \\
\text { supplementa- } \\
\text { tion LIST } \\
\text { exercise } \\
\text { program }\end{array}$ & $\begin{array}{c}\text { CG }(n=11) \\
3.2 \mathrm{~g} / \text { day } \\
6 \text { weeks. }\end{array}$ & $\begin{array}{l}\text { Post-LIST RTD } \\
\text { reduction }\end{array}$ & NR & $\begin{array}{c}\downarrow \text { RTD decline in } \\
\text { both groups } \\
\text { without } \\
\text { significant } \\
\text { difference }\end{array}$ & Level 3 & 1 \\
\hline
\end{tabular}


Table 3. Cont

\begin{tabular}{|c|c|c|c|c|c|c|c|c|c|c|c|}
\hline Authors & Study Design & $\begin{array}{l}\text { Population } \\
\text { Number } \\
\text { Groups } \\
\text { Gender } \\
\text { Mean Age }\end{array}$ & $\begin{array}{l}\text { Intervention } \\
\text { Matrix }\end{array}$ & $\begin{array}{l}\text { TG size } \\
\text { Dosage } \\
\text { Frequency } \\
\text { Duration }\end{array}$ & $\begin{array}{l}\text { Control } \\
\text { Matrix }\end{array}$ & $\begin{array}{l}\text { CG Size } \\
\text { Dosage } \\
\text { Frequency } \\
\text { Duration }\end{array}$ & $\begin{array}{l}\text { Endpoints/Data } \\
\text { Collection Tools }\end{array}$ & $\begin{array}{l}\text { Adverse } \\
\text { Events }\end{array}$ & Results & ОСЕВМ & EPHPP \\
\hline $\begin{array}{c}\text { Kotsis, Y., et al. } \\
\text { (2018) [41] }\end{array}$ & RCT, DB, PC & $\begin{array}{l}\text { Soccer players } \\
n=22 ; \text { mean } \\
\text { age NR } \\
n=4 \text { excluded } \\
\text { from the } \\
\text { analysis }\end{array}$ & $\begin{array}{c}\text { Commercial } \\
\text { BC } \\
378 \mathrm{Kcal}, 67 \mathrm{~g} \\
\text { protein, } 17 \mathrm{~g} \\
\text { carbohydrates } \\
\text { and } 4.7 \mathrm{~g} \text { fat } \\
\text { per } 100 \mathrm{~g} \\
\text { Pre and post } \\
\text { supplementa- } \\
\text { tion LIST } \\
\text { exercise } \\
\text { program }\end{array}$ & $\begin{array}{c}\text { TG }(n=10) \\
3.2 \mathrm{~g} / \text { day } \\
6 \text { weeks }+ \\
4 \text { days }\end{array}$ & $\begin{array}{l}\text { Commercial } \\
\text { whey protein } \\
369 \mathrm{Kcal}, 90 \mathrm{~g} \\
\text { protein, } 1 \mathrm{~g} \\
\text { carbohydrates } \\
\text { and } 0.5 \mathrm{~g} \mathrm{fat} \\
\text { per } 100 \mathrm{~g} \\
\text { Pre and post } \\
\text { supplementa- } \\
\text { tion LIST } \\
\text { exercise } \\
\text { program }\end{array}$ & $\begin{array}{c}\text { CG }(n=8) \\
3.2 \mathrm{~g} / \text { day } \\
6 \text { weeks }+ \\
4 \text { days }\end{array}$ & $\begin{array}{c}\text { EIMD: MIVC, SQJ, } \\
\text { CMJ, PMS, } \\
\text { biochemical } \\
\text { parameters }\end{array}$ & NR & $\begin{array}{c}\text { } \text { SQJ, CRP, CK, } \\
\text { IL-6 recovery. } \\
\text { No significant } \\
\text { differences on } \\
\text { MIVC, CMJ, } \\
\text { PMS and } \\
\text { other outcome }\end{array}$ & Level 2 & 1 \\
\hline $\begin{array}{c}\text { March, D. S., } \\
\text { et al. (2018) } \\
\text { [43] }\end{array}$ & $\begin{array}{c}\text { RCT, DB, PC, } \\
\text { CO }\end{array}$ & $\begin{array}{c}\text { Regularly } \\
\text { exercising } \\
\text { males } \\
n=12 ; \text { mean } \\
\text { age } 26 \text { ys } \\
\text { Colostrum } \\
(n=12) \text { vs. } \\
\text { Placebo } \\
(n=12) \\
\text { CO after } \\
2 \text { weeks of } \\
\text { washout }\end{array}$ & $\begin{array}{l}\text { BC (Neovite } \\
\text { UK, London) }\end{array}$ & $\begin{array}{c}\text { TG }(n=12): \\
\text { BC } 20 \mathrm{~g} / \text { day } \\
\text { for } 14 \text { days } \\
\text { Exercise } \\
\text { program } 70 \% \\
\text { aerobic } \\
\text { capacity for } \\
1 \mathrm{~h}\end{array}$ & & $\begin{array}{l}\text { CG }(n=12): \\
\text { Isoener- } \\
\text { getic/Isomacronu } \\
\text { placebo for } \\
14 \text { days } \\
\text { Exercise } \\
\text { program } 70 \% \\
\text { aerobic } \\
\text { capacity for } \\
1 \mathrm{~h}\end{array}$ & $\begin{array}{c}\text { PE: } \\
\text { Exercise-induced } \\
\text { trieintestinal cell } \\
\text { damage (I-FABP) } \\
\text { SE: (1) Bacterial } \\
\text { translocation } \\
\text { (plasmatic } \\
\text { bacterial DNA) } \\
\text { (2) Other physical } \\
\text { parameters }\end{array}$ & NR & $\begin{array}{c}\downarrow \text { I-FABP plasma } \\
\text { concentration } \\
\text { after exercise } \\
\text { No effects } \\
\text { Bacterial DNA } \\
\text { plasmatic } \\
\text { concentration } \\
\text { No significant } \\
\text { differences on } \\
\text { other outcome }\end{array}$ & Level 2 & 1 \\
\hline $\begin{array}{c}\text { Morrison, S. } \\
\text { A., et al. (2014) } \\
\text { [45] }\end{array}$ & $\begin{array}{c}\text { RCT, DB, PC, } \\
\text { CO }\end{array}$ & $\begin{array}{c}\text { Trained and } \\
\text { Untrained } \\
\text { males } n=28 \\
\text { (14 trained, } \\
14 \text { untrained) } \\
\text { Colostrum } \\
(n=14) \text { vs. } \\
\text { Placebo }(n=14) \\
n=13 \\
\text { (6 untrained, } \\
7 \text { trained) lost } \\
\text { and excluded } \\
\text { from the } \\
\text { analysis } \\
\text { CO time NR }\end{array}$ & $\begin{array}{c}\text { BC } \\
\text { (Hokitika, } \\
\text { New Zealand) } \\
\text { protein, } 58.2 \% \\
\mathrm{~m} / \mathrm{m} \text {; fat, } 1.4 \% \\
\mathrm{~m} / \mathrm{m} ; \text { lactose, } \\
\text { 29.3\% } \mathrm{m} / \mathrm{m} ; \\
\text { and IgG, } \\
15.3 \% \text {. } \\
\text { Before } 90 \mathrm{~min} \\
\text { multi-mode } \\
\text { exercise } \\
\text { session }\end{array}$ & $\begin{array}{c}\text { TG }(n=7 \\
\text { trained, } n=8 \\
\text { untrained): BC } \\
1.7 \mathrm{~g} / \mathrm{kg} / \text { day } \\
\text { for } 7 \text { days }\end{array}$ & $\begin{array}{l}\text { Corn flour } \\
\text { placebo }\end{array}$ & $\begin{array}{c}\text { CG }(n=7 \\
\text { trained, } n=8 \\
\text { untrained): } \\
\text { corn flour } \\
\text { placebo for } \\
7 \text { days before } \\
90 \text { min } \\
\text { multi-mode } \\
\text { exercise } \\
\text { session }\end{array}$ & $\begin{array}{c}\text { PE: GI } \\
\text { permeability } \\
\text { (Double sugar } \\
\text { model, I-FABP) } \\
\text { SE: (1) cytokine } \\
\text { level and other } \\
\text { blood parameters } \\
\text { (2) thermal and } \\
\text { cardiovascular } \\
\text { measures } \\
\text { (3) Other } \\
\text { parameters }\end{array}$ & No AEs & $\begin{array}{l}\uparrow \text { I-FABP in } \\
\text { trained group } \\
\text { No significant } \\
\text { differences on } \\
\text { other outcome }\end{array}$ & Level 3 & 1 \\
\hline
\end{tabular}


Table 3. Cont.

\begin{tabular}{|c|c|c|c|c|c|c|c|c|c|c|c|}
\hline Authors & Study Design & $\begin{array}{l}\text { Population } \\
\text { Number } \\
\text { Groups } \\
\text { Gender } \\
\text { Mean Age }\end{array}$ & $\begin{array}{l}\text { Intervention } \\
\text { Matrix }\end{array}$ & $\begin{array}{l}\text { TG size } \\
\text { Dosage } \\
\text { Frequency } \\
\text { Duration }\end{array}$ & $\begin{array}{l}\text { Control } \\
\text { Matrix }\end{array}$ & $\begin{array}{l}\text { CG Size } \\
\text { Dosage } \\
\text { Frequency } \\
\text { Duration }\end{array}$ & $\begin{array}{l}\text { Endpoints/Data } \\
\text { Collection Tools }\end{array}$ & $\begin{array}{c}\text { Adverse } \\
\text { Events }\end{array}$ & Results & OCEBM & EPHPP \\
\hline $\begin{array}{c}\text { Morrison, S. } \\
\text { A., et al. (2013) } \\
\text { [44] }\end{array}$ & $\begin{array}{c}\text { RCT, DB, PC, } \\
\text { CO }\end{array}$ & $\begin{array}{c}\text { Healthy males } \\
n=15 \\
\text { ( } 7 \text { highly-fit, } \\
8 \text { moderately- } \\
\text { fit); mean age } \\
22 \text { ys }\end{array}$ & $\begin{array}{c}\text { BC } \\
\text { (Hokitika, } \\
\text { New Zealand) } \\
\text { protein, } \\
58.2 \% \mathrm{~m} / \mathrm{m} ; \\
\text { fat, } 1.4 \% \mathrm{~m} / \mathrm{m} ; \\
\text { lactose, } 29.3 \% \\
\mathrm{~m} / \mathrm{m} \text {; and IgG, } \\
15.3 \% \text {. } \\
\text { Before } 90 \mathrm{~min} \\
\text { multi-mode } \\
\text { exercise } \\
\text { session }\end{array}$ & $\begin{array}{c}\text { TG }(n=7 \\
\text { highly fit, } n=8 \\
\text { moderately- } \\
\text { fit }) \\
1.7 \mathrm{~g} / \mathrm{kg} / \text { day } \\
7 \text { days }\end{array}$ & $\begin{array}{l}\text { Corn flour } \\
\text { placebo }\end{array}$ & $\begin{array}{c}\mathrm{CG}(n=7 \\
\text { highly fit, } n=8 \\
\text { moderately fit) } \\
1.77 \mathrm{~g} / \mathrm{kg} / \text { day } \\
7 \text { days }\end{array}$ & $\begin{array}{c}\text { PE: (1) BBB } \\
\text { permeability } \\
\text { (S100ß protein, } \\
\text { cerebral } \\
\text { oxygenation) } \\
\text { (2) Cognitive } \\
\text { function (Stroop } \\
\text { test and } \\
\text { perceptions) } \\
\text { SE: (1) thermal and } \\
\text { cardiovascular } \\
\text { measures } \\
\text { (2) Other } \\
\text { parameters }\end{array}$ & NR & $\begin{array}{l}\text { No effects on } \\
\text { BBB, cognitive } \\
\text { and physical } \\
\text { performance }\end{array}$ & Level 3 & 1 \\
\hline $\begin{array}{c}\text { Shing, C. M., } \\
\text { et al. (2013) } \\
{[46]}\end{array}$ & $\begin{array}{l}\text { RCT, DB, PC } \\
\text { Pilot study }\end{array}$ & $\begin{array}{c}\text { Highly- } \\
\text { trained males } \\
n=10 ; \text { mean } \\
\text { age NR } \\
\text { CPC }(n=4) \text { vs. } \\
\text { WPC placebo } \\
\quad(n=6)\end{array}$ & $\begin{array}{c}\text { Intact }^{\circledR} \text { bovine } \\
\text { CPC (Numico } \\
\text { Research } \\
\text { Australia Pty } \\
\text { Ltd., South } \\
\text { Australia) } \\
\text { before and } \\
\text { during a } \\
5 \text { days cycling } \\
\text { race }\end{array}$ & $\begin{array}{c}\text { TG }(n=4) \\
10 \mathrm{~g} / \text { day for } \\
8 \text { weeks }+ \\
5 \text { days }\end{array}$ & $\begin{array}{c}\text { Whey protein } \\
\text { concentrate } \\
\text { (Alacen }{ }^{\circledR} 80^{\prime \prime} \\
\text { Fonterra } \\
\text { Co-op Group } \\
\text { Limited, } \\
\text { Auckland, } \\
\text { New Zealand) } \\
\text { before and } \\
\text { during a } \\
5 \text { days cycling } \\
\text { race }\end{array}$ & $\begin{array}{c}\text { CG }(n=6) \\
10 \mathrm{~g} / \text { day for } \\
8 \text { weeks }+ \\
5 \text { days }\end{array}$ & $\begin{array}{c}\text { PE: hormonal } \\
\text { (salivary hormones } \\
\text { level), immune } \\
\text { (salivary IgA) and } \\
\text { autonomic } \\
\text { (parasympathetic } \\
\text { indices of HRV) } \\
\text { response } \\
\text { SE: Mood profile } \\
\text { (POMS) }\end{array}$ & NR & $\begin{array}{c}\uparrow \text { testosterone } \\
\text { concentration } \\
\text { maintenance } \\
\uparrow \text { cortisol } \\
\text { concentration } \\
\text { before the race } \\
\uparrow \text { parasympa- } \\
\text { thetic indices of } \\
\text { HRV } \\
\text { No significant } \\
\text { differences on } \\
\text { cortisol } \\
\text { concentration } \\
\text { during race, } \\
\text { POMS and } \\
\text { salivary IgA } \\
\text { concentration }\end{array}$ & Level 3 & 1 \\
\hline
\end{tabular}

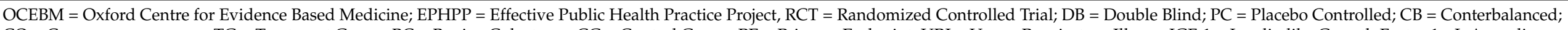

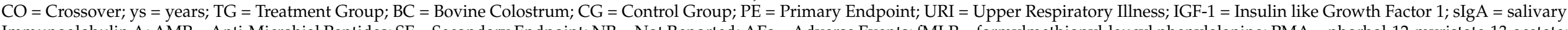

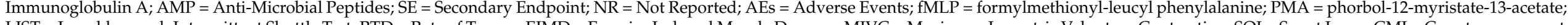

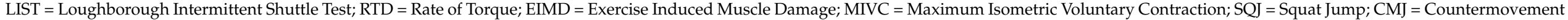

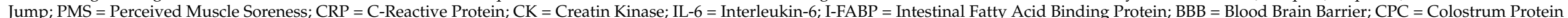
Concentrate; WPC $=$ Whey Protein Concentrate; HRV = Hearth Rate Variability; POMS = Profile Of Mood States questionnaire. Symbols: $\uparrow=$ increased; $\downarrow=$ decreased. 
Table 4. BC as dietary supplement in the pediatric and preterm infants.

\begin{tabular}{|c|c|c|c|c|c|c|c|c|c|c|c|}
\hline Authors & Study Design & $\begin{array}{l}\text { Population } \\
\text { Number } \\
\text { Groups } \\
\text { Gender } \\
\text { Mean Age }\end{array}$ & $\begin{array}{l}\text { Intervention } \\
\text { Matrix }\end{array}$ & $\begin{array}{l}\text { TG size } \\
\text { Dosage } \\
\text { Frequency } \\
\text { Duration }\end{array}$ & $\begin{array}{l}\text { Control } \\
\text { Matrix }\end{array}$ & $\begin{array}{l}\text { CG Size } \\
\text { Dosage } \\
\text { Frequency } \\
\text { Duration }\end{array}$ & $\begin{array}{l}\text { Endpoints/Data } \\
\text { Collection Tools }\end{array}$ & $\begin{array}{c}\text { Adverse } \\
\text { Events }\end{array}$ & Results & OCEBM & EPHPP \\
\hline $\begin{array}{c}\text { Aunsholt, L., } \\
\text { et al. (2014) } \\
\text { [47] }\end{array}$ & $\begin{array}{c}\text { RCT, DB, PC, } \\
\text { CO, PS }\end{array}$ & $\begin{array}{c}\text { Children with } \\
\text { SBS } \\
n=9 \text { (4 females, } \\
5 \text { males); median } \\
\text { age } 39 \text { months } \\
\text { CO after } 4 \text { weeks } \\
\text { washout }\end{array}$ & $\begin{array}{l}\text { “Fresh" BC } \\
\text { from } 15 \\
\text { different cows } \\
\text { (Danish } \\
\text { Holstein) } \\
\text { within the first } \\
24 \mathrm{~h} \text { after } \\
\text { calving }\end{array}$ & $\begin{array}{c}\text { TG }(n=9) \\
20 \% \text { of BFR }\end{array}$ & $\begin{array}{l}\text { Mixed milk, } \\
\text { cream, and } \\
\text { whey protein }\end{array}$ & $\begin{array}{c}\text { CG }(n=9) \\
20 \% \text { of BFR }\end{array}$ & $\begin{array}{l}\text { PE: Nutrients and } \\
\text { Fluid balances } \\
\text { SE: } \\
\text { Anthropometric, } \\
\text { knemometry, } \\
\text { biological } \\
\text { parameters }\end{array}$ & NR & $\begin{array}{c}\text { No significant } \\
\text { differences in } \\
\text { intestinal energy } \\
\text { and wet weight } \\
\text { absorption, } \\
\text { knemometry, } \\
\text { IGF-1, IGF-BP3 } \\
\text { levels }\end{array}$ & Level 2 & 1 \\
\hline $\begin{array}{c}\text { Balachandran, } \\
\text { B., et al. (2016) } \\
\text { [48] }\end{array}$ & $\begin{array}{c}\text { RCT, DB, PC, } \\
\text { PS }\end{array}$ & $\begin{array}{c}\text { VLBW Neonates } \\
n=86 ; \\
\text { chronological } \\
\text { age }<96 \mathrm{~h} \\
\text { Colostrum } \\
(n=43) \text { vs. } \\
\text { Placebo }(n=43)\end{array}$ & $\begin{array}{c}\text { BC } \\
\text { Pedimmune }^{\circledR} \\
\text { (Mumbai, }^{{ }^{\circledR}} \\
\text { India) }\end{array}$ & $\begin{array}{c}\text { TG }(n=43) \\
1.2-2.0 \mathrm{~g} / \text { dose } \\
+ \text { feeding } \\
4 \text { times a day } \\
21 \text { days }\end{array}$ & $\begin{array}{l}\text { Equal dose } \\
\text { placebo (not } \\
\text { specified) }\end{array}$ & $\begin{array}{c}\text { CG }(n=43) \\
1.2-2.0 \mathrm{~g} / \mathrm{die}+ \\
\text { feeding } \\
4 \text { times a day } \\
21 \text { days }\end{array}$ & $\begin{array}{c}\text { PE: NECSE: sepsis, } \\
\text { mortality and stool } \\
\text { interleukin-6 (IL-6) } \\
\text { levels }\end{array}$ & No AEs & $\begin{array}{c}\text { No significant } \\
\text { differences in } \\
\text { NEC, sepsis and } \\
\text { mortality. } \\
\uparrow I L-6 \text { and } \\
\text { radiological } \\
\text { features of NEC } \\
\text { in TG }\end{array}$ & Level 2 & 1 \\
\hline $\begin{array}{l}\text { Meinich Juhl, } \\
\text { S., et al. (2018) } \\
\text { [50] }\end{array}$ & $\mathrm{RCT}, \mathrm{OL}, \mathrm{PS}$ & $\begin{array}{c}\text { NPI } \\
n=40 ; \\
\text { gestational age } \\
\text { 27-32 weeks } \\
\text { Country } \\
\text { stratification } \\
\text { (China- } \\
\text { Denmark) }\end{array}$ & $\begin{array}{c}\text { Unmodified } \\
\text { intact BC } \\
\text { powder } \\
\text { (ColoDan }{ }^{\circledR} ; \\
\text { Gesten, } \\
\text { Denmark) as } \\
\text { supplement of } \\
\text { MM, DM or IF }\end{array}$ & $\begin{array}{c}\text { TG }(n=21) \\
\text { max } \\
4.5 \mathrm{~g} / \mathrm{kg} / \text { day } \\
10-14 \text { days }\end{array}$ & $\begin{array}{c}\text { Standard } \\
\text { feeding with } \\
\text { MM, DM or IF }\end{array}$ & $\begin{array}{l}\text { CG }(n=19) \\
10-14 \text { days }\end{array}$ & $\begin{array}{l}\text { PE: Tolerability } \\
\text { and safety } \\
\text { SE: nutritional } \\
\text { outcomes }\end{array}$ & $\begin{array}{c}1 \text { death for } \\
\text { NEC not } \\
\text { related to BC } \\
\text { intake } \\
\text { Late onset } \\
\text { sepsis (2 TG, } \\
1 \text { CG), } \\
\text { pulmonary } \\
\text { dysplasia } \\
(1 \mathrm{TG}), \text { ROP (2 } \\
\text { TG), metabolic } \\
\text { acidosis (2 TG) } \\
\text { No significant } \\
\text { differences }\end{array}$ & $\begin{array}{c}\uparrow \text { protein intake } \\
\text { in TG (China } \\
\text { group) } \\
\uparrow \text { in TG } \\
\text { Temporary } \\
\text { elevation in } \\
\text { plasmatic } \\
\text { tyrosine levels } \\
\text { on day } 7 \\
\text { No significant } \\
\text { differences on } \\
\text { dietary } \\
\text { intolerances and } \\
\text { other outcomes }\end{array}$ & Level 3 & 2 \\
\hline
\end{tabular}


Table 4. Cont.

\begin{tabular}{|c|c|c|c|c|c|c|c|c|c|c|c|}
\hline Authors & Study Design & $\begin{array}{c}\text { Population } \\
\text { Number } \\
\text { Groups } \\
\text { Gender } \\
\text { Mean Age }\end{array}$ & $\begin{array}{l}\text { Intervention } \\
\text { Matrix }\end{array}$ & $\begin{array}{l}\text { TG size } \\
\text { Dosage } \\
\text { Frequency } \\
\text { Duration }\end{array}$ & $\begin{array}{l}\text { Control } \\
\text { Matrix }\end{array}$ & $\begin{array}{l}\text { CG Size } \\
\text { Dosage } \\
\text { Frequency } \\
\text { Duration }\end{array}$ & $\begin{array}{l}\text { Endpoints/Data } \\
\text { Collection Tools }\end{array}$ & $\begin{array}{l}\text { Adverse } \\
\text { Events }\end{array}$ & Results & OCEBM & ЕРНРP \\
\hline $\begin{array}{l}\text { Patiroğlu, T. } \\
\text { and M. } \\
\text { Kondolot } \\
\text { (2013) [51] }\end{array}$ & RCT, DB, PC & $\begin{array}{l}\text { IgA deficient } \\
\text { paediatrics with } \\
\quad \text { viral URI } \\
n=31 ; \text { median } \\
\text { age } 8.5 \text { ys; } \\
n=18 \text { males and } \\
13 \text { females } \\
\text { Colostrum } \\
(n=16) \text { vs. } \\
\text { Placebo }(n=15)\end{array}$ & $\begin{array}{l}\text { BC sucking } \\
\text { tablet that } \\
\text { contains } 14 \mathrm{mg} \\
\text { of colostrum } \\
\text { and } 2.2 \mathrm{mg} \text { of } \\
\text { lysozyme } \\
\text { (Igazym }^{\circledR} ; \\
\text { Vejle, } \\
\text { Denmark) }\end{array}$ & $\begin{array}{c}\text { TG }(n=16) \\
3 \text { times a day } \\
1 \text { week }\end{array}$ & $\begin{array}{c}\text { placebo } \\
\text { sucking tablets }\end{array}$ & $\begin{array}{c}\text { CG }(n=15) \\
3 \text { times a day } \\
\text { for } 1 \text { week }\end{array}$ & $\begin{array}{l}\text { PE: sIgA } \\
\text { SE: Infection } \\
\text { severity }\end{array}$ & $\begin{array}{l}\text { No AEs } \\
1 \text { patient } \\
\text { included } \\
2 \text { times and } 1 \\
\text { included } 3 \\
\text { time for } \\
\text { different } \\
\text { infections }\end{array}$ & $\begin{array}{l}\text { No significant } \\
\text { differences in } \\
\text { slgA secretion } \\
\text { \infection } \\
\text { severity score in } \\
\text { BC after } 1 \text { week }\end{array}$ & Level 2 & 1 \\
\hline $\begin{array}{l}\text { Rathe, M., et al. } \\
\text { (2020) [52] }\end{array}$ & $\begin{array}{l}\text { RCT, DB, PC } \\
\text { Multicentre } \\
\text { study }\end{array}$ & $\begin{array}{c}\text { Pediatrics with } \\
\text { ALL } \\
n=62, \text { aged } \\
1-18 \text { ys } \\
n=32 \text { males and } \\
30 \text { females } \\
\text { Colostrum } \\
(n=30) \text { vs. } \\
\text { Placebo }(n=32)\end{array}$ & $\begin{array}{c}\text { Intact, } \\
\text { spray-dried } \\
\text { BC powder } \\
\text { (Gesten, } \\
\left.\text { Denmark }^{\circledR}\right) .\end{array}$ & $\begin{array}{c}\text { TG }(n=30) \\
0.5-1 \mathrm{~g} / \mathrm{kg} / \text { day } \\
4 \text { weeks }\end{array}$ & $\begin{array}{l}\text { Isocaloric } \\
\text { placebo. } \\
\text { whole-milk } \\
\text { powder } \\
\text { enriched with } \\
\text { whey protein } \\
\text { isolate powder }\end{array}$ & $\begin{array}{c}\text { CG }(n=32) \\
4 \text { weeks }\end{array}$ & $\begin{array}{l}\text { PE: fever level and } \\
\text { duration.SE: CRP } \\
\text { levels, neutrophil } \\
\text { count, bacteraemia } \\
\text { or fungaemia } \\
\text { episodes, } \\
\text { treatment delay, } \\
\text { mucositis severity, } \\
\text { PROs on } \\
\text { chemotherapy } \\
\text { toxicity, } \\
\text { compliance }\end{array}$ & No AEs & $\begin{array}{c}\downarrow \text { Peak severity } \\
\text { of oral mucositis } \\
\text { No significant } \\
\text { differences on } \\
\text { PE and other SE, } \\
\text { low compliance } \\
\text { reported }\end{array}$ & Level 2 & 1 \\
\hline $\begin{array}{l}\text { Saad, K., et al. } \\
\text { (2016) [53] }\end{array}$ & $\begin{array}{l}\text { Cohort } \\
\text { Prospective } \\
\text { Multicentric }\end{array}$ & $\begin{array}{c}\text { Children with } \\
\text { recurrent acute } \\
\text { URI or diarrhea } \\
\text { due to infection } \\
n=160 ; \\
\text { aged } 1-6 \text { ys. } \\
n=81 \text { males and } \\
79 \text { females }\end{array}$ & $\begin{array}{c}\text { ImmuGuard }^{\circledR}, \\
\text { sachets } \\
\text { (London, } \\
\text { England) }\end{array}$ & $\begin{array}{c}\text { TG } n=160 \\
3 \mathrm{~g} / \text { sachet } \\
4 \text { weeks. }\end{array}$ & No CG & No CG & $\begin{array}{l}\text { URI or diarrhea } \\
\text { episodes and } \\
\text { frequency of } \\
\text { hospitalizations } \\
\text { Follow up period } \\
\quad 24 \text { weeks }\end{array}$ & $\begin{array}{l}\text { Mild transient } \\
\text { AEs reported } \\
\text { in } 12 \text { patients: } \\
\text { Skin rush (9), } \\
\text { itching (1), and } \\
\text { diarrhea (2). } \\
6 \text { patients } \\
\text { discontinued } \\
\text { the BC } \\
\text { treatment. }\end{array}$ & $\begin{array}{c}\downarrow \text { Infection } \\
\text { episodes at } 2 \\
\text { and } 6 \text { months } \\
\downarrow \text { Hospitaliza- } \\
\text { tions }\end{array}$ & Level 3 & 3 \\
\hline
\end{tabular}

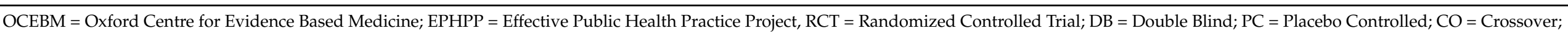

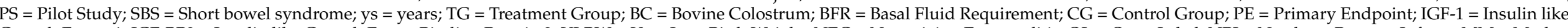

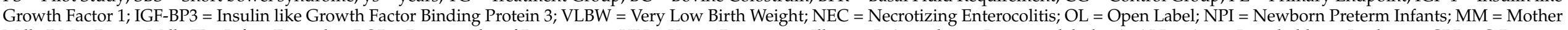

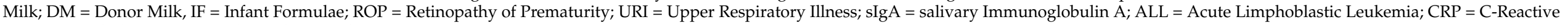
Protein. Symbols: $\uparrow=$ increased; $\downarrow$ = decreased. 
Table 5. BC as dietary supplement in healthy older adults.

\begin{tabular}{|c|c|c|c|c|c|c|c|c|c|c|c|}
\hline Authors & Study Design & $\begin{array}{c}\text { Population } \\
\text { Number } \\
\text { Groups } \\
\text { Gender } \\
\text { Mean Age }\end{array}$ & $\begin{array}{l}\text { Intervention } \\
\text { Matrix }\end{array}$ & $\begin{array}{l}\text { TG Size } \\
\text { Dosage } \\
\text { Frequency } \\
\text { Duration }\end{array}$ & $\begin{array}{l}\text { Control } \\
\text { Matrix }\end{array}$ & $\begin{array}{l}\text { CG Size } \\
\text { Dosage } \\
\text { Frequency } \\
\text { Duration }\end{array}$ & $\begin{array}{l}\text { Endpoints/Data } \\
\text { Collection Tools }\end{array}$ & $\begin{array}{l}\text { Adverse } \\
\text { Events }\end{array}$ & Results & OCEBM & EPHPP \\
\hline
\end{tabular}

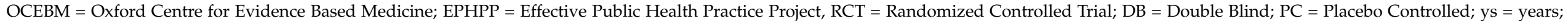

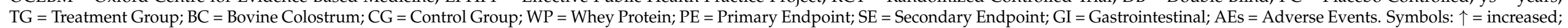

$\downarrow=$ decreased.

Table 6. BC administration in critically ill patients.

\begin{tabular}{|c|c|c|c|c|c|c|c|c|c|c|c|}
\hline Authors & Study Design & $\begin{array}{c}\text { Population } \\
\text { Number } \\
\text { Groups } \\
\text { Gender } \\
\text { Mean Age }\end{array}$ & $\begin{array}{l}\text { Intervention } \\
\text { Matrix }\end{array}$ & $\begin{array}{l}\text { TG Size } \\
\text { Dosage } \\
\text { Frequency } \\
\text { Duration }\end{array}$ & $\begin{array}{l}\text { Control } \\
\text { Matrix }\end{array}$ & $\begin{array}{l}\text { CG Size } \\
\text { Dosage } \\
\text { Frequency } \\
\text { Duration }\end{array}$ & $\begin{array}{l}\text { Endpoints/Data } \\
\text { Collection Tools }\end{array}$ & $\begin{array}{l}\text { Adverse } \\
\text { Events }\end{array}$ & Results & OCEBM & ЕРНPP \\
\hline $\begin{array}{c}\text { Eslamian, G., } \\
\text { et al. (2019) } \\
\text { [55] }\end{array}$ & RCT, DB, PC & $\begin{array}{c}\text { ICU patients } \\
n=70 ; \text { mean age } \\
62 \text { ys } \\
\text { Colostrum } \\
(n=35) \text { vs. } \\
\text { Placebo }(n=35) \\
n=8 \text { not } \\
\text { included in the } \\
\text { analysis }\end{array}$ & $\begin{array}{c}\text { Neovite }{ }^{\circledR} \\
\text { (London, UK). }\end{array}$ & $\begin{array}{l}\text { TG }(n=32): \\
\text { BC } 20 \mathrm{~g} / \text { day }+ \\
\text { enteral feeding } \\
\text { for } 10 \text { days }\end{array}$ & $\begin{array}{c}\text { ENTERA } \\
\text { Meal }^{\circledR} ; \\
\text { (Tehran, Iran) }\end{array}$ & $\begin{array}{c}\text { CG }(n=30): \\
\text { Isocaloric } \\
\text { maltodextrin }+ \\
\text { enteral feeding } \\
\text { for } 10 \text { days }\end{array}$ & $\begin{array}{l}\text { PE: Intestinal } \\
\text { permeability } \\
\text { (plasmatic } \\
\text { endotoxin and } \\
\text { zonulin) } \\
\text { SE: mortality, LOS, } \\
\text { GI complications }\end{array}$ & No AEs & $\begin{array}{c}\downarrow \text { Plasmatic } \\
\text { endotoxin and } \\
\text { zonulin } \\
\text { concentrations at } \\
\text { day } 10 \text {. } \\
\downarrow \text { Diarrhea } \\
\text { No significant } \\
\text { differences in } \\
\text { other } \\
\text { comparisons }\end{array}$ & Level 2 & 1 \\
\hline
\end{tabular}

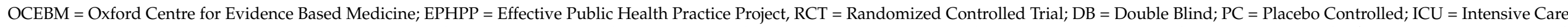

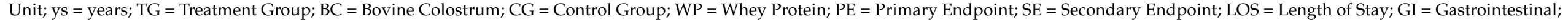

AEs $=$ Adverse Events. Symbol: $\downarrow=$ decreased. 
Table 7. Abstracts on BC clinical applications.

\begin{tabular}{|c|c|c|c|c|c|c|c|c|}
\hline Authors & Study Design & $\begin{array}{l}\text { Population } \\
\text { Number } \\
\text { Groups } \\
\text { Gender } \\
\text { Mean Age }\end{array}$ & $\begin{array}{l}\text { Interventions } \\
\text { Dosage } \\
\text { Frequency } \\
\text { Duration }\end{array}$ & $\begin{array}{l}\text { Control } \\
\text { Dosage } \\
\text { Frequency } \\
\text { Duration }\end{array}$ & $\begin{array}{c}\text { Endpoints } \\
\text { Data Collection } \\
\text { Tools }\end{array}$ & Adverse Events & Results & OCEBM \\
\hline $\begin{array}{l}\text { Caysido et al. } \\
\text { (2017) [56] }\end{array}$ & RCT, DB, PC & $\begin{array}{c}\text { Pediatrics } \\
\text { undergoing } \\
\text { chemotherapy for } \\
\text { ALL } \\
n=21 \text {; aged } 6 \\
\text { months to } 18 \text { ys }\end{array}$ & $\begin{array}{c}\text { TG }(n=\mathrm{NR}) \text { : BC } \\
\text { twice a day for a } \\
\text { week from the first } \\
\text { day of } \\
\text { chemotherapy }\end{array}$ & $\begin{array}{c}\text { CG }(n=\text { NR) } \\
\text { Placebo twice a } \\
\text { day for a week } \\
\text { from the first day } \\
\text { of chemotherapy }\end{array}$ & $\begin{array}{l}\text { PE: neutropenia } \\
\text { (CBC, ANC) }\end{array}$ & No AEs & $\begin{array}{l}\uparrow \text { ANC, WBC and } \\
\text { PLT blood levels }\end{array}$ & Level 5 \\
\hline $\begin{array}{l}\text { Barakat, S., et al. } \\
\quad(2019) b \text { [57] }\end{array}$ & RCT, DB, PC & $\begin{array}{c}\text { Pediatrics with } \\
\text { acute diarrhea } \\
n=160 ; \\
\text { aged } 6 \text { months to } \\
2 \text { ys }\end{array}$ & $\begin{array}{c}\text { TG }(n=80) \\
\text { BC } 3 \text { g/day for } \\
1 \text { week }\end{array}$ & $\begin{array}{c}\text { CG }(n=80) \\
\text { placebo for } 1 \text { week }\end{array}$ & $\begin{array}{c}\text { PE: } n^{\circ} \text { of patients } \\
\text { with diarrhea after } \\
72 \mathrm{~h}\end{array}$ & NR & $\begin{array}{c}\text { Diarrhea stopped } \\
\text { in } 65 \% \text { of TG vs. } \\
95 \% \text { of CG after } 72 \\
\text { h }\end{array}$ & Level 5 \\
\hline $\begin{array}{l}\text { Donowitz, J., et al. } \\
\text { (2019) [58] }\end{array}$ & RCT, PC & $\begin{array}{l}\text { Infants (income } \\
\text { country) } \\
n=\mathrm{NR} ; 6 \text { to } \\
9 \text { months }\end{array}$ & $\begin{array}{c}\text { TG }(n=\mathrm{NR}) \\
7 \mathrm{~g} \text { of PTM202 } \\
\text { twice a day } \\
30 \text { days }\end{array}$ & $\begin{array}{c}\text { CG }(n=\mathrm{NR}) \\
\text { micronutrient } \\
\text { sprinkles twice } \\
\text { a day } \\
30 \text { days }\end{array}$ & $\begin{array}{l}\text { PE: EED (fecal } \\
\text { MPO, fecal Reg 1B, } \\
\text { serum CRP, serum } \\
\text { sCD14, and L:M }\end{array}$ & NR & $\begin{array}{c}\downarrow \text { fecal MPO and } \\
\text { reg1B } \\
\text { No significant } \\
\text { differences in } \\
\text { other parameters }\end{array}$ & Level 5 \\
\hline $\begin{array}{c}\text { Oloroso-Chavez, } \\
\text { K., et al. (2017) } \\
\text { [59] }\end{array}$ & $\begin{array}{c}\mathrm{RCT} \\
\text { Subgroup analysis }\end{array}$ & $\begin{array}{c}\text { Pediatrics with } \\
\text { respiratory allergies } \\
n=38 ; \text { aged } 7 \text { to } \\
18 \text { ys }\end{array}$ & $\begin{array}{l}\text { TG }(n=19) \\
\text { BC } 1000 \text { mg day } \\
3 \text { months. }\end{array}$ & $\begin{array}{c}\text { CG }(n=19) \\
\text { Placebo } 1000 \mathrm{mg} \\
\text { day } \\
3 \text { months }\end{array}$ & $\begin{array}{l}\text { PE: Symptoms } \\
\text { improvement } \\
\text { (TNSS, ACT, CASI } \\
\text { and pulmonary } \\
\text { function test) }\end{array}$ & NR & $\begin{array}{c}\downarrow \text { nasal congestion } \\
\text { (TNSS) and lung } \\
\text { function in } \\
\text { monosensitized } \\
\text { subjects } \\
\uparrow \text { ACT and CASI } \\
\text { scores in } \\
\text { polysensitized } \\
\text { subjects }\end{array}$ & Level 5 \\
\hline
\end{tabular}


Table 8. Systematic reviews on BC clinical applications.

\begin{tabular}{|c|c|c|c|c|c|c|c|}
\hline Authors & Study Design & $\begin{array}{c}\text { n of Paper Included } \\
\text { Heterogeneity } \\
\text { n of Participants }\end{array}$ & $\begin{array}{c}\text { Bovine Colostrum Effects } \\
\text { Measured }\end{array}$ & Evidence & Adverse Events & ОСЕВМ & AMSTAR 2 \\
\hline Rathe, M., et al. (2014) [21] & Systematic review & $\begin{array}{l}49 \text { record covering } \\
51 \text { studies } \\
\text { High heterogeneity: } \\
\text { settings, methodologies, } \\
\text { treatment/placebos } \\
\text { preparations and dosages, } \\
\text { population, diseases, } \\
\text { endpoints and outcomes } \\
2326 \text { participants }\end{array}$ & $\begin{array}{ll}\text { - } & \text { NSAID and surgery } \\
\text { induced GI toxicity } \\
\text { (2 RCT- } n=122 \\
\text { participants) } \\
\text { HIV associated diarrhea } \\
\text { and immunosuppression } \\
\text { (1 CSS, 3 OBS, } 1 \text { RT- } \\
n=182 \text { participants }) \\
\text { Sports nutrition and } \\
\text { exercise (12 RCT, } 1 \text { NRT, } 1 \\
\text { OBS- } n=370 \text { participants) } \\
\text { Immune functions in sport } \\
\text { and exercise }(9 \text { RCT; } \\
1 \text { NRT- } n=244 \\
\text { participants) } \\
\text { Infection and immune } \\
\text { responses }(10 \text { RCT, } \\
1 \text { OBS }-n=1090 \\
\text { participants) } \\
\text { SBS }(2 \text { RCT- } n=21 \\
\text { participants) } \\
\text { Growth and metabolic } \\
\text { Disorders }(1 \text { RCT, } 1 \text { OBS- } \\
n=138 \text { participants }) \\
\text { Juvenile idiopathic } \\
\text { arthritis } 1 \text { RCT- } \\
n=30 \text { participants) } \\
\text { Chronic pain syndrome } \\
\text { and irritable bowel } \\
\text { syndrome }(1 \text { RCT, } \\
1 \text { CSS - } n=114 \\
\text { participants) }\end{array}$ & $\begin{array}{ll}\text { - } & \text { Evidence suggests that BC } \\
\text { protect GI tract from NSAID } \\
\text { (short period) induced injuries } \\
\text { Evidence suggests that BC may } \\
\text { reduce microbial translocation } \\
\text { across the gut mucosa in } \\
\text { patients undergoing abdominal } \\
\text { surgery } \\
\text { Evidence suggests that BC can } \\
\text { effectively ameliorate } \\
\text { HIV-associated diarrhea } \\
\text { Contradictory evidence are } \\
\text { shown on the effects of bovine } \\
\text { colostrum on sports } \\
\text { performance, body } \\
\text { composition, and nutrient } \\
\text { absorption } \\
\text { Not conclusive evidence are } \\
\text { available on the effects of } \\
\text { colostrum on immunity } \\
\text { Evidence suggests that BC } \\
\text { triggers immunological events } \\
\text { followed by systemic ones } \\
\text { No evidence of a marked effect } \\
\text { on intestinal function has been } \\
\text { documented in SBS patient } \\
\text { Inconclusive evidence of } \\
\text { benefit of BC on thrive failures } \\
\text { and on metabolic control of } \\
\text { type II diabetes } \\
\text { Evidence of no effect of BC } \\
\text { supplementation on clinical } \\
\text { outcomes } \\
\text { Evidence of no significant effect } \\
\text { of BC on irritable bowel } \\
\text { syndrome }\end{array}$ & $\begin{array}{l}\text { No serious AEs } \\
\text { Mild/moderate AEs were } \\
\text { reported: unpleasant taste, } \\
\text { nausea, flatulence, } \\
\text { diarrhea, skin rash, and } \\
\text { unspecified abdominal } \\
\text { discomfort. } \\
\text { Nine studies reported an } \\
\text { absence of side effects. }\end{array}$ & Level 1 & HIGH \\
\hline
\end{tabular}

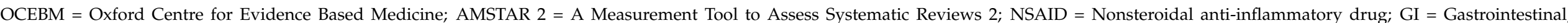

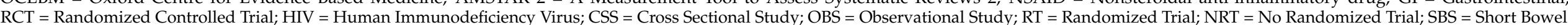
Syndrome; BC = Bovine Colostrum; AEs = Adverse Events. 
Table 9. CCRBT bias assessment.

\begin{tabular}{|c|c|c|c|c|c|c|c|}
\hline & 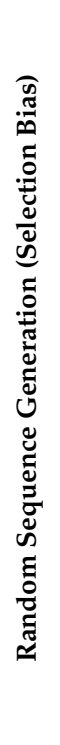 & 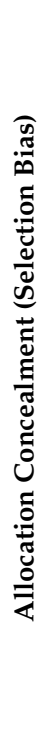 & 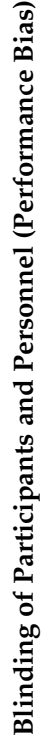 & 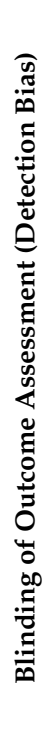 & 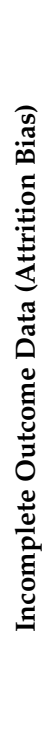 & 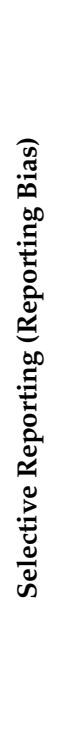 & 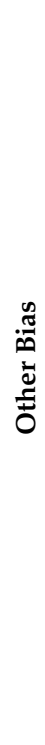 \\
\hline Nappi, R. E., et al. (2016) & + & + & - & ? & + & ? & + \\
\hline Jones, A. W., et al. (2014) & $?$ & $?$ & + & + & + & + & + \\
\hline Davison G., et al. (2019) & $?$ & $?$ & + & + & + & $?$ & + \\
\hline Halasa, M., et al. (2017) & + & + & + & + & + & $?$ & $?$ \\
\hline Jones, A. W., et al. (2019) & + & + & + & + & + & ? & ? \\
\hline Jones A.W., et al. (2015) & $?$ & $?$ & + & + & + & + & + \\
\hline Kotsis, Y., et al. (2019) & + & + & + & + & $?$ & $?$ & + \\
\hline Kotsis, Y., et al. (2018) & + & + & + & + & + & + & $?$ \\
\hline March, D. S., et al. (2018) & + & + & + & + & + & + & + \\
\hline Morrison S. A., et al. (2014) & $?$ & $?$ & + & + & - & $?$ & $?$ \\
\hline Morrison, S. A., et al. (2013) & $?$ & $?$ & + & + & + & $?$ & - \\
\hline Shing, C. M., et al. (2013) & + & $?$ & + & $?$ & + & $?$ & + \\
\hline Aunsholt, L., et al. (2014) & + & $?$ & + & + & + & + & + \\
\hline Balachandran, B., et al. (2016) & + & + & + & $?$ & + & $?$ & $?$ \\
\hline Barakat et al. (2019) & + & + & + & + & + & + & + \\
\hline Meinich Juhl, S., et al. (2018) & + & + & - & - & + & $?$ & $?$ \\
\hline Patıroğlu, T. and M. Kondolot (2013) & + & $?$ & + & $?$ & + & + & + \\
\hline Rathe, M., et al. (2020) & + & + & + & + & + & ? & + \\
\hline Duff, W. R., et al. (2014) & + & + & + & + & + & + & + \\
\hline Eslamian, G., et al. (2019) & + & + & + & + & + & + & + \\
\hline
\end{tabular}

\subsection{BC Topical Applications in Uro-Gynecology Setting}

BC was used as component of topical ointments in the treatment of different vaginal conditions (Table 2). Nappi et al. [33] studied the efficacy of a BC-based gel cream (Monurelle Biogel ${ }^{\circledR}$ ) on Vaginal Dryness (VD) of 95 women randomized in two groups, patients in the Treatment Group (TG; $n=48$ ) received BC gel during the intermenstrual period (23 days) versus no treatment in the Control Group (CG; $n=47)$. Clinical success was defined as the reduction of the vaginal discomfort of at least 1 point on a 5 points Verbal Rating Scale after the treatment period. Vaginal symptoms were significantly decreased in TG $(92.7 \%$ vs. $63.6 \% ; p=0.002)$. Assessing vaginal health, significant improvements of the Vaginal Health Index (VHI) scores were obtained in TG than CG (4.4 \pm 2.6 vs. $1.2 \pm 1.9$; $p<0.001)$, as well as the sexual function evaluated by the Female Sexual Function Index (FSFI) $(p<0.032)$. No differences were on Female Sexual Distress Scale-revised (FSDS-R) scores $(p=0.16)$. The same product was used daily for 12 weeks in a large retrospective cohort $(n=172)$ of women (mean age 61 years) with Vulvo-Vaginal Atrophy (VVA) [34]. In this study VHI mean scores were improved from $12.5 \pm 3.67$ at baseline to $19.3 \pm 3.49$ after treatment $(p<0.001)$, FSFI scores from $21.64 \pm 2.99$ to $28.16 \pm 1.93(p<0.001)$ and FSDS scores decreased from $20.52 \pm 5.90$ to $8.15 \pm 4.18(p<0.001)$. The effectiveness of BC-based 
vaginal tablets (Ginedie ${ }^{\circledR}$ ) on Cervical Intraepithelial Neoplasia low grade lesions (CIN1) caused by the Human Papilloma Virus (HPV) was retrospectively assessed in a large group ( $n=256)$ of patients (mean age 38 years) [35]. After 6 months of treatment (twice a week night time) $71 \%$ of cases were histologically negative while in $27 \%$ CIN1 histology was confirmed and in $2 \%$ the evolution to CIN2 was observed.

\subsection{BC as Dietary Supplement in the Sporting Population}

Eleven double blinded placebo controlled randomized trials reports were included in this category [36-46]. One of them included three RCTs [39] and 3 trials adopted a crossover design $[39,43,45]$. These trials recruited only male subjects $(n=289$; range $10-57$ participants; mean age range $22-51$ years) at various level of training including regularly/recreationally trained people (7 RCTs) [36-39,43-45] and highly trained ones such as cyclists, soccer players and professional fighters (4 RCT) [40-42,46]. Desiccated BC and placebo formulations were administered to the participants within beverages. The dosages varied among studies from 1 to $60 \mathrm{~g}$ /day as well as the exposition duration was variable from $4.5 \mathrm{~h}$ to 12 weeks. The majority of control groups $(n=5)$ received isoenergetic and isomacronutrient placebo [36-39,43]; whey proteins $(n=4)[40-42,46]$ and corn flour (2) were also used. In 3 trials, BC and placebo were administered as dietary supplement $[36,37,40]$ without other variables modification while in 8 studies were associated to various exercise programs. These programs differed between them for intensity and duration [38,39,41-46]. In one study BC was associated to desiccated banana [40] and in another one was administered as colostrum protein concentrate [46]. A wide heterogeneity was present regarding assessed outcomes. The efficacy of BC preventing Upper Respiratory Illnesses (URI) were evaluated by one trial [36] (53 participants; mean age 51 years) that reported significant difference between groups on URI incidence $(0.4 \pm 0.7$ vs. $0.8 \pm 0.7$; $p=0.03$ ) after 12 weeks of treatment and a significantly lower proportion of URI duration (days) $(0.05$ vs. $0.09 ; p<0.001)$. One study including 3 RCTs reported findings on safety [39] measuring IGF-1 blood level variations after assumption of $40 \mathrm{~g}$ of BC during $4.5 \mathrm{~h}$ of moderate exercises ( $n=16$ participants), and then after $4(n=20)$ and 12 weeks $(n=57)$ of $20 \mathrm{~g} /$ day supplementation. No significant changes (per study group and per time) were reported. The gut permeability was assessed by 3 RCTs $[40,43,45]$. BC was more effective than placebo reducing sugar absorption $(p=0.01)$ and zonulin stool concentration $(p=0.03)$ after 20 days of supplementation in 16 athletic males during competitive period [40]. In a crossover trial $(n=20)$, Intestinal Fatty Acid Binding Protein (I-FABP) plasmatic level increasing were significantly less $(p=0.015$ and $p=0.019$ at the end and $1 \mathrm{~h}$ after exercises respectively) in treatment group of regularly exercised males undergoing study specific exercise program (heat condition) after 14 days of BC supplementation (20 g/day) [43]. The same outcome was evaluated in another RCT recruiting 57 trained and untrained subjects supported for 7 days with $B C$ or placebo at $1.7 \mathrm{~g} / \mathrm{kg} /$ day [45]. Immediately after the study exercise, I-FABP blood levels significantly increased than baseline in both groups $(p<0.001)$, but higher values were in trained group compared with untrained one ( $p=0.006)$. Two RCTs by Jones et al. [37,38] ( $n=34, n=20$, respectively) evaluated the effects of BC on immune response. BC supplementation ( $20 \mathrm{~g} /$ day) for 58 days blunted immune response attenuation due to physical activity and increasing immune sensitivity at $24 \mathrm{~h}(p<0.001)$ and at $48 \mathrm{~h}(p=0.023)$. No significant effects on in-vivo immune responsiveness, IGF-1 blood levels, immune cell counts [37]. Improved attenuation of the decline in formyl-Methionyl-Leucyl-Phenylalanine (fMLP) stimulated oxidative burst response in a model of exercise-induced immune dysfunction after 4 weeks of $\mathrm{BC}$ administration (20 g/day) was showed in a little placebo controlled trial (10 subjects per arm) $(p<0.05)$. No significant effects on other receptors or on mucosal barrier function were observed [38]. The effects of $\mathrm{BC}$ ( $3.2 \mathrm{~g} /$ day for 6 weeks) on muscular health and performance maintaining were studied in two trials [41,42] involving 44 soccer players (22 per RCT) undergone the Loughborough Intermittent Shuttle Test (LIST). The decline of rate of torque was reduced in both groups (intervention and placebo) without significant difference as well as no 
difference resulted by maximum isometric voluntary contraction, countermovement jump and perceived muscle soreness comparisons. Some beneficial effects were showed on faster recovery of squat jump performance and of some biochemical parameters. The effects of $\mathrm{BC}$ on blood brain barrier (BBB) permeability and cognitive function (CF) were assessed by one trial [44] recruiting 15 trained and untrained men in a crossover design study. $\mathrm{BC}$ and placebo were administered for 7 days at $1.7 \mathrm{~g} / \mathrm{kg} /$ day before a $90 \mathrm{~min}$ exercise program in heat conditions. No significant results were obtained. Significant differences were showed in a pilot study by Shing et al. [46] on hormonal and autonomic response of cyclists assigned to BC $(n=4)$ supplementation $(10 \mathrm{~g} /$ day over 8 weeks of colostrum protein concentrate) than placebo (whey protein concentrate) $(n=6)$. Table 3 summarize further information on this category.

\subsection{BC as Dietary Supplement in Pediatrics and Preterm Infants}

Six RCTs and 1 OS were in this category, all of them exploring the effects of BC administration as dietary supplement (Table 4) [47-53]. Five RCTs were double blinded placebo controlled [47-49,51,52] while 1 was controlled versus standard practice without blinding [50]. The studies recruited 548 patients (RCTs $n=388$, OS $n=160$ ), 290 males and 258 females including toddlers [47,53], newborns [48], infants and toddlers [49], preterm newborns [50], any children [51,52]. Seven different diseased populations were involved in the 7 studies: Short Bowel Syndrome (SBS) [47], Very Low Birth Weight (VLBW) newborns [48], acute diarrhea and vomit [49], preterm newborns [50], IgA deficient children [51], Acute Lymphoblastic Leukemia (ALL) [52], recurrent acute URI or diarrhea [53]. BC was administered within beverage (various powder formulas) in 5 studies [48-50,52,53], sucking tablets in 1 [51] and as "fresh product" in the study by Aunsholt et al. [47] It was administered at various dosages ranging from $14 \mathrm{mg}$ to $4.5 \mathrm{~g} / \mathrm{kg} /$ day and following different protocols (from 1 to 12 weeks, from 1 to 4 time per day) based on ageing, underlying diseases, nutritional needs and study protocols. Aunsholt et al. studied the supplementation with BC (versus milk mixed matrix) of $20 \%$ of the Basal Fluid Requirement (BFR) in children $(n=9)$ affected by SBS. A crossover designed RCT was performed. The lack of significant differences among groups on energy $(p=1)$ and wet weight absorption $(p=0.93)$ suggested that $\mathrm{BC}$ dietary supplementation did not improve intestinal function in these patients [47]. Balachandran et al. evaluated the efficacy of BC in a pilot study comparing BC $(1.2-2.0 \mathrm{~g} / \mathrm{dose}$, 4 time/day for 21 days) and placebo in 86 VLBW neonates. No significant differences on incidence of Necrotizing Enterocolitis (NEC), sepsis and mortality were found between groups ( $p=0.4, p=0.4, p=1$ respectively). However, a trend toward a higher incidence of NEC in BC group was showed (9.3\% vs. $2.1 \%, \mathrm{RR}=4.31,95 \% \mathrm{CI}=0.42-105.82)$ [48]. An RCT recruited 160 children (6 months- 2 years) with acute diarrhea [49] randomizing them to receive $\mathrm{BC}$ ( $3 \mathrm{~g} /$ day for 1 week) or placebo in addition to standard therapy. After $48 \mathrm{~h}$, the BC group had a significantly lower frequency of vomiting, diarrhea, fever and Vesikari scoring compared with the placebo group $(p<0.001, p=0.001, p<0.001, p<0.001$, respectively). Significant results on number of children with diarrhea were reported after $72 \mathrm{~h}$ and 7 days ( $35 \%$ vs. $94 \%, p<0.001 ; 0 \%$ vs. $12.5 \%, p=0.001$ respectively) suggesting beneficial effects of BC supplementation in this population. Tolerability and safety of $\mathrm{BC}$ ( $\max 4.4 \mathrm{~g} / \mathrm{kg} /$ day for 10-14 days) in addition to standard feeding (mother milk, donor milk or infant formula) versus standard feeding alone were studied on 40 preterm neonates [50]. A number of Adverse Events (AEs) without significant differences between groups were reported by this study, including 1 death (NEC not related to colostrum supplementation), 3 sepsis, 1 bronchopulmonary dysplasia, 2 retinopathies, 4 intraventricular hemorrhages. No significant differences in dietary intolerances were reported. No significant difference in salivary IgA secretion was reported in a RCT involving $31 \operatorname{IgA}$ deficient patients who received BC $14 \mathrm{mg}+$ lysozyme $2.2 \mathrm{mg}$ sucking tablets 3 time per day for 1 week [51]. Rathe et al. in a multicenter RCT recruited 62 pediatric patients affected by ALL (age range 1-18 years) [52]. Patients were randomized to receive a BC or an isocaloric placebo $(0.5-1 \mathrm{~g} / \mathrm{kg} /$ day) during 4 weeks of chemotherapy treatment. Data on fever level 
and duration, bacteriemia episodes, mucositis severity and biochemical parameters were collected. Severity of oral mucositis (peaks) resulted significantly reduced in BC group $(p=0.02)$; however, BC did not show any effects on fever, infections and other outcomes. A large cohort prospective multicenter study by Saad et al. enrolled 160 children ( 81 males, 79 females) between 1 and 6 years with recurrent infectious URI or diarrhea [53]. The cohort was supplemented for 4 weeks with $3 \mathrm{~g} /$ day of BC. Reduction of infection episodes were observed at 2 and 6 months follow up (mean $8.6 \pm 5.1$ baseline vs. $5.5 \pm 1.2$ after 2 months; $p<0.001$ vs. $5.7 \pm 1.6$ after 6 months; $p<0.001$ ) and the reduction of hospitalization frequency was reported $(p<0.001)$. Further details on included study of this category were in Table 4.

\subsection{BC as Dietary Supplement in Healthy Older Adults}

Only one paper was found in this field. The RCT by Duff et al. compared the effects of $\mathrm{BC}$ versus whey protein complex ( $60 \mathrm{~g} /$ day for 8 weeks) randomizing 40 healthy elderlies (mean age 59 years, 15 males and 25 females) undergone a study specific exercise training program. Outcome such as body composition, strength, muscle thickness, bone resorption, cognitive function and biochemical exams were assessed in the trial. In the treatment group, the leg press strength was significantly higher $(24 \pm 29 \mathrm{~kg}, p<0.01)$ while urinary $\mathrm{N}$-telopeptides (Ntx), that is considered a marker of bone resorption, was significantly reduced $(p<0.05)$. No difference on other outcomes were found [54].

\subsection{BC Administration in Critically Ill Patients}

A double blinded placebo controlled randomized trial was identified that assessed colostrum intake in critically ill patients ( $n=70$, mean age 62 years). A dosage of $20 \mathrm{~g} /$ day of $\mathrm{BC}$ or placebo (isocaloric maltodextrin) were added to enteral feeding for 10 days [55]. Intestinal permeability was the primary end-point and it was assessed measuring plasmatic endotoxin and zonulin concentrations. Plasmatic endotoxin concentration and zonulin levels were significantly lower in BC group after 10 days of treatment $(p<0.05$ and $p<0.001$, respectively). In addition, in BC group the incidence of diarrhea was lower $(p=0.02)$.

\subsection{Abstracts on BC Clinical Applications}

Four of 28 papers included in this review were only available as abstracts. All of them [56-59] were placebo controlled RCTs and concerned the pediatric setting. They showed $\mathrm{BC}$ benefits increasing neutrophil account in LLA patients who underwent chemotherapy [56]; decreasing acute diarrhea in infants after $72 \mathrm{~h} \mathrm{[57],} \mathrm{reducing} \mathrm{enteric} \mathrm{inflammation}$ in exposed children [58] and reducing nasal congestion promoting pulmonary function in children affected by respiratory allergies [59]. The authors of this review thought it was important to include these abstracts because they might highlight new research options or confirm results of other studies. However, it was not possible to perform a quality assessment of these records due to poor available information.

\subsection{Systematic Reviews on BC Clinical Applications}

Table 8 summarize the results provided by the last systematic review on clinical applications of BC (enteral intake only).

\section{Discussion}

Three included studies considered different BC topical applications in uro-gynecology setting [33-35]. VD was often associated with a genitourinary syndrome, leading to sexual dysfunction and poor quality of life of affected women; the vaginal gel formulation $\left(\right.$ Monurelle Bio-gel ${ }^{\circledR}$ ) used in two studies included in this review $[33,34]$ showed characteristics similar to the physiological vaginal secretions creating a conducive to natural lubrication environment. Considering the high patients' compliance and the lack of AEs reported by these studies, very encouraging results were found on vaginal and sexual health as well as urinary symptoms. Despite these findings were available by one RCT 
alone without blinding strategies, the reviewer considered robust its results attributing high level of evidence supporting the use of this formulae to reduce VD symptoms in young women (OCEBM and EPHPP level 1 evidence). In elderly women this approach seemed to be effective managing VD due to vaginal atrophic issues [34]. However, weak evidence (Table 2) may be provided in this population and these findings should be confirmed by well-designed RCTs. The immune-stimulatory and nutritive functions of topical application of BC could have a positive effect in the management of CIN1 lesions [35]. However, the level of evidence provided on this topic (OCEBM 4 and EPHPP 3) should be improved with randomized controlled studies. The humectant, moisturizing, re-epithelizing, antioxidant and immune-stimulant activities of $\mathrm{BC}$ were effective improving vaginal health and reducing sexual distress in women affected by VD conditions. Furthermore, the topical use of BC appeared safe and inexpensive. Further well-designed studies such as randomized controlled trials should be conducted to increase the level of evidence of the results on CIN1 and to explore the effects of BC topical applications in other conditions.

Bovine colostrum was used as dietary supplement in the sporting population. The studies included explored the benefits of $B C$ as natural sporting nutritional supplement on subjects' well-being and physical performances [36-46]. Jones et al. [36], used BC as a nutritional supplement to boost immunity and reduce the risk of URI in athletes. The study showed that BC supplementation limited salivary bacterial load and reduced URI more significantly than placebo supplements. Some selection bias-related concerns (Table 9) and the characteristics of the sample (only males) limited the level of evidence provided by this study. As reported by Rathe et al. in the systematic review included here [21], the relationship among physical exercise intensiveness and URI development depends on many factors and is not well recognized, yet. In agreement with the previous findings, not conclusive evidence were provided by our review due to study shortage, heterogeneity and risk of bias. Further well designed RCTs are warranted in this population. The safety of BC use during physical activity was explored. IGF-1 plasmatic levels did not increase during the short and long periods after the administration of standard doses of $\mathrm{BC}$ associated to training programs [39]. Intestinal permeability may change due to various factors such as inflammation or heat stress produced by intensive physical activity [38]. The safety and effectiveness of BC supplementation intake on athletes' health were demonstrated in another placebo-controlled comparison where the reduction of the intestinal permeability was highlighted measuring zonulin stool concentration and sugar absorption [40]. However, some doubts on reporting bias and the very little sample size did not allow to provide strong evidence on these findings. Intestinal fatty acid binding protein (I-FABP) was considered a marker of gut heat-related permeability changes in subjects undergone intensive physical exercises [60]. The effectiveness of BC supplementation reducing its plasmatic level increasing after intensive activity was detected in a placebo controlled RCT included in this review [43]. However, despite there was a main effect in the treatment arm, this trial reported no statistically significant effect on bacterial plasmatic DNA concentration. These results were in contrast with the study by Morrison et al. [45] that showed how prolonged exertion in hot environment enough to produce heat stress increased gastrointestinal permeability independently by subjects' level of training. I-FABP was higher in colostrum group and more in trained people than untrained ones in this study. Further studies are mandatory in order to provide further knowledge on factors influencing intestinal damage and its permeability changes during exercise with or without colostrum supplementation. Weak evidence of no effects were provided evaluating the efficacy of BC on blood brain barrier permeability and cognitive function [44]. Despite evidence of effects were showed on some outcomes, inconclusive findings were available on $B C$ effects on immune system responsiveness during intensive exercises (neutrophil response and innate immunity) $[37,38]$ as well as on $B C$ effects on physical performances and muscle damaging [41,42] due to the lack of response on other outcomes considered in these studies, the large use of surrogate endpoints and their very small sample sizes. Interesting results was showed examining the effects of $\mathrm{BC}$ on participants' hormonal 
profile that could have impact in recovery improvement and fatigue reduction; however, these results must be carefully considered due to very small sample size of the pilot study included [46]. An agreement would be needed in scientific community defining the endpoints to be included in well-designed randomized controlled trials to better explore the effects of $\mathrm{BC}$ in athlete populations.

The seven studies included in this review exploring the use of BC as dietary supplement in pediatric population and preterm infants were very heterogeneous in terms of the target population, outcomes, and study designs. Short Bowel Syndrome (SBS) causes nutrients and fluids malabsorption and may lead to severe consequences such as necrotizing enterocolitis, volvulus or gastroschisis [61]. The pilot RCT included in this review [47] hypothesized that the $\mathrm{BC}$ high concentration of peptide hormones, immunoglobulins, macro and micronutrients could implement intestinal absorption in SBS children. However, confirming Rathe and colleagues findings [21], evidence of no effects on intestinal absorption and metabolic balance of $\mathrm{BC}$ supplementation in this population was reported by our review. Although a protective effect of $\mathrm{BC}$ on gastrointestinal mucosa was suggested, there were evidence of no effects of $B C$ supplementation on neutropenic fever, antibiotic drugs utilization and bacteriemia incidence in Acute Lymphoblastic Leukemia pediatric patients undergoing chemotherapy. A large cohort study by Saad et al. [53] showed that oral administered BC prophylaxis in children affected by recurrent Upper Respiratory Tract Infections (URTI) and diarrhea reduced both infection and hospitalization rates maintaining a protective effect in the long time period. The direction of these results was confirmed by two double-blind RCTs with placebo where BC was effective reducing severity of URTI affecting IgA deficient patients [51] and decreasing frequency and severity of both vomiting and diarrhea in infants with gastrointestinal infection diseases [49]. The authors hypothesized that the positive effect of $\mathrm{BC}$ may be traced to its high concentration of immunoglobulins (IgG, IgM, IgE, and IgD), lactoferrin and lysozyme. These findings should be still managed carefully in relation to patients' clinical condition and ageing due to controversial results achieved on $\mathrm{BC}$ supplementation in frailest populations of this category (preterm and VLBW infants) [48,50]. In these two settings BC administration for mothers' milk supplementation resulted a feasible option for protein intake enhancing without increasing feeding intolerance and without any side effects, apparently [50]. However, a number of AEs was reported in preterm infant population (equally distributed among groups) [50] and a trend of increased frequencies of necrotizing enterocolitis and sepsis were observed in treatment group [48]. These findings might due to the frailty of the studied populations (AEs) as well as to the production process of the colostrum-based product used [48]. It is known that industrial processed formula products may increase necrotizing enterocolitis and sepsis in this population [50]. Despite BC supplementation in pediatrics suggested protective effects on respiratory infectious diseases and other conditions related symptoms, its role preventing gastrointestinal issues or supporting preterm infants remain controversial. Further studies are needed in order to provide new evidence on $\mathrm{BC}$ administration as dietary supplement in pediatric populations (such as those with cancer) and to clarify the effects of its different preparations.

$\mathrm{BC}$ was used as dietary supplement in healthy older adults in a single double blind $\mathrm{RCT}$, where $\mathrm{BC}$ intake outcomes were assessed during a specific resistance training and compared with those of whey proteins administered as placebo. $\mathrm{BC}$ was effective increasing legs strength (OCEBM level 2 and EPHPP level 1 evidence) and reducing bone resorption. No significant results were found on inflammation, upper body performances and body composition. These findings were interesting in order to promote further investigations on BC effects on bone health in this population [54].

The effects on gastrointestinal permeability of early enteral bovine colostrum supplementation versus placebo were investigated in intensive care unit (ICU) setting [55]. Significant reductions of plasmatic concentrations of both zonulin and endotoxin were found (high level of evidence), meaning safe effect reducing intestinal permeability in these 
patients. In addition, positive results were found on length of ICU stay and diarrhea. No effects were found on mortality, sepsis and other gastrointestinal outcomes.

Two systematic reviews were published in the time period considered. The review by Blair et al. [62] was aimed to evaluate the benefits of both milk and BC as dietary supplements in the healthy adult population $\geq 35$ years old. However, this review included only one study on BC already selected in our review, then the review was excluded as not pertinent. The review by Rathe et al. [21] included 51 reports including RCTs, observational and case series studies published until March 2013. The review assessed the effects of BC enteral supplementation. Evidence of BC's benefits was reported on patients' intestinal tract protection from non-steroidal anti-inflammatory drugs (NSAID) induced injuries, and on bacterial translocation through gut barrier in patients undergoing abdominal surgery as well as on immuno-compromised patients' (Human Immunodeficiency Virus) diarrhea control. In addition, inconclusive evidence were showed on growth or metabolic issues and idiopathic arthritis. We could not update these findings in our review due to the lack of further studies. Despite was clear that BC's activity may triggers bowel immunological events that provides systemic ones, and its biological effects on intestinal permeability and upper respiratory tract health were explored suggesting a role of $B C$ supplementation in URI prevention and intestinal infections treatment in both adult and pediatric populations; the interaction mechanisms of $\mathrm{BC}$ components with the immune system are largely underexplored. This suggests the need of pre-clinical (in-vitro and in-vivo) studies to better understand the process that influences its effects.

\section{Conclusions}

This review highlighted multiple clinical applications of BC and confirmed some general benefits on intestinal and respiratory recovery in absence of adverse effects. BC seemed to promote immune system enhancing and modulating local and systemic responses in various clinical and not clinical conditions. However, the studies' heterogeneity regarding included populations, sample sizes, intervention and control protocols, and outcomes did not allow to perform meta-analyses. Moreover, the risk of biases and the large use of surrogate endpoints in the studies included did not consent to provide strong evidence on its use in any situation. Further well-designed studies are needed to support the administration of BC in adult, pediatric, clinical and not clinical settings. Pre-clinical studies should be performed to improve knowledge on BC effects.

Author Contributions: Conceptualization, M.G. and M.P.; methodology, M.G.D.M.; software, A.F., M.T.C. and S.N.; validation, M.G.D.M. and S.B.; formal analysis, A.F. and S.B.; investigation, M.G. and A.C.; resources, L.D.; data curation, A.F.; writing-original draft preparation, L.D. and S.B.; writingreview and editing, S.B.; visualization, A.F.; supervision, M.G., M.P.; project administration, M.G., M.G.D.M. and M.P. All authors have read and agreed to the published version of the manuscript.

Funding: This research received no external funding.

Institutional Review Board Statement: Not applicable.

Informed Consent Statement: Not applicable.

Data Availability Statement: No new data were created or analyzed in this study. Data sharing is not applicable to this article.

Acknowledgments: All authors thank to Lucia Mariani for the English language editing. Thanks to Massimo Costantini and to the AUSL-IRCCS of Reggio Emilia Health Professions Directorate for their support.

Conflicts of Interest: The authors declare no conflict of interest. 


\section{References}

1. Bagwe, S.; Tharappel, L.J.P.; Kaur, G.; Buttar, H.S. Bovine Colostrum: An Emerging Nutraceutical. J. Complement Integr. Med. 2015, 12, 175-185. [CrossRef]

2. Parrish, D.B.; Wise, G.H.; Hughes, J.S. Properties of the Colostrum of the Dairy Cow. I Tocopherol Levels in the Colostrum and in the Early Milk1. J. Dairy Sci. 1947, 30, 849-860. [CrossRef]

3. Parrish, D.B.; Wise, G.H.; Hughes, J.S.; Atkeson, F.W. Properties of the Colostrum of the Dairy Cow. II. Effect of Prepartal Rations upon the Nitrogenous Constituents1. J. Dairy Sci. 1948, 31, 889-895. [CrossRef]

4. Parrish, D.B.; Wise, G.H.; Hughes, J.S. Properties of the Colostrum of the Dairy Cow. IV. Effect of Form of Vitamin A and of Tocopherol Supplements on Concentrations of Vitamin A and Carotenoids1. J. Dairy Sci. 1949, 32, 458-464. [CrossRef]

5. Moody, E.G.; Wise, G.H.; Parrish, D.B.; Atkeson, F.W. Properties of the Colostrum of the Dairy Cow. VI. Creaming and Rate of Flow1. J. Dairy Sci. 1951, 34, 106-115. [CrossRef]

6. McGrath, B.A.; Fox, P.F.; McSweeney, P.L.H.; Kelly, A.L. Composition and Properties of Bovine Colostrum: A Review. Dairy Sci. Technol. 2016, 96, 133-158. [CrossRef]

7. Carrillo, A.E.; Koutedakis, Y.; Flouris, A.D. Exercise and Exposure to Heat Following Bovine Colostrum Supplementation: A Review of Gastrointestinal and Immune Function. Cell Mol. Biol. 2013, 59, 84-88.

8. Stelwagen, K.; Carpenter, E.; Haigh, B.; Hodgkinson, A.; Wheeler, T.T. Immune Components of Bovine Colostrum and Milk. J Anim. Sci. 2009, 87, 3-9. [CrossRef]

9. Lönnerdal, B. Bioactive Proteins in Breast Milk. J. Paediatr. Child Health 2013, 49, 1-7. [CrossRef]

10. Filipescu, I.E.; Leonardi, L.; Menchetti, L.; Guelfi, G.; Traina, G.; Casagrande-Proietti, P.; Piro, F.; Quattrone, A.; Barbato, O.; Brecchia, G. Preventive Effects of Bovine Colostrum Supplementation in TNBS-Induced Colitis in Mice. PLoS ONE 2018, 13, e0202929. [CrossRef]

11. Dice Nail, C. The Immunoprotective Properties of Bovine Colostrum: A Review. Nutr. Perspect. J. Counc. Nutr. 2016, 39, 23-30.

12. Gopal, P.K.; Gill, H.S. Oligosaccharides and Glycoconjugates in Bovine Milk and Colostrum. Br. J. Nutr. 2000, 84 (Suppl. 1), S69-S74. [CrossRef]

13. Menchetti, L.; Traina, G.; Tomasello, G.; Casagrande-Proietti, P.; Leonardi, L.; Barbato, O.; Brecchia, G. Potential Benefits of Colostrum in Gastrointestinal Diseases. Front. Biosci. 2016, 8, 331-351. [CrossRef]

14. Marnila, P.; Korohnen, H. Colostrum. Encyclopedia of Dairy Sciences; Academic Press: Cambridge, MA, USA, 2002.

15. Tsioulpas, A.; Grandison, A.S.; Lewis, M.J. Changes in Physical Properties of Bovine Milk from the Colostrum Period to Early Lactation. J. Dairy Sci. 2007, 90, 5012-5017. [CrossRef]

16. Barrington, G.M.; Besser, T.E.; Davis, W.C.; Gay, C.C.; Reeves, J.J.; McFadden, T.B. Expression of Immunoglobulin G1 Receptors by Bovine Mammary Epithelial Cells and Mammary Leukocytes. J. Dairy Sci. 1997, 80, 86-93. [CrossRef]

17. Butler, J.E. The Occurrence of Immunoglobulin Fragments, Two Types of Lactoferrin and a Lactoferrin-IgG2 Complex in Bovine Colostral and Milk Whey. Biochim. Biophys. Acta Protein Struct. 1973, 295, 341-351. [CrossRef]

18. Pakkanen, R.; Aalto, J. Growth Factors and Antimicrobial Factors of Bovine Colostrum. Int. Dairy J. 1997, 7, 285-297. [CrossRef]

19. Apodaca, G.; Katz, L.A.; Mostov, K.E. Receptor-Mediated Transcytosis of IgA in MDCK Cells Is via Apical Recycling Endosomes. J. Cell Biol. 1994, 125, 67-86. [CrossRef] [PubMed]

20. Phalipon, A.; Cardona, A.; Kraehenbuhl, J.-P.; Edelman, L.; Sansonetti, P.J.; Corthésy, B. Secretory Component: A New Role in Secretory IgA-Mediated Immune Exclusion In Vivo. Immunity 2002, 17, 107-115. [CrossRef]

21. Rathe, M.; Müller, K.; Sangild, P.T.; Husby, S. Clinical Applications of Bovine Colostrum Therapy: A Systematic Review. Nutr. Rev. 2014, 72, 237-254. [CrossRef]

22. van Hooijdonk, A.C.M.; Kussendrager, K.D.; Steijns, J.M. In Vivo Antimicrobial and Antiviral Activity of Components in Bovine Milk and Colostrum Involved in Non-Specific Defence. Br. J. Nutr. 2000, 84, 127-134. [CrossRef]

23. Hurley, W.L.; Theil, P.K. Perspectives on Immunoglobulins in Colostrum and Milk. Nutrients 2011, 3, 442-474. [CrossRef]

24. Sarker, S.A.; Casswall, T.H.; Mahalanabis, D.; Alam, N.H.; Albert, M.J.; Brüssow, H.; Fuchs, G.J.; Hammerström, L. Successful Treatment of Rotavirus Diarrhea in Children with Immunoglobulin from Immunized Bovine Colostrum. Pediatric Infect. Dis. J. 1998, 17, 1149-1154. [CrossRef] [PubMed]

25. Doillon, C.J.; Lehance, F.; Bordeleau, L.J.; Laplante-Campbell, M.P.; Drouin, R. Modulatory Effect of a Complex Fraction Derived from Colostrum on Fibroblast Contractibility and Consequences on Repair Tissue. Int. Wound J. 2011, 8, 280-290. [CrossRef] [PubMed]

26. Aalto, J.U.; Jalkanen, M.T.; Jalonen, H.G.; Kanttinen, A.P.; Laato, M.K.; Pakkanen, R.A. Method for the Improvement of Wound Healing and Compositions Therefore. WIPO (PCT) WO1995000155 A1, 5 January 1995.

27. Główka, N.; Woźniewicz, M. Potential Use of Colostrum Bovinum Supplementation in Athletes-A Review. Acta Sci. Pol. Technol. Aliment. 2019, 18, 115-123. [CrossRef]

28. Gingerich, D.A.; McPhillips, C.A. Analytical Approach to Determination of Safety of Milk Ingredients from Hyperimmunized Cows. Regul. Toxicol. Pharmacol. 2005, 41, 102-112. [CrossRef]

29. Liberati, A.; Altman, D.G.; Tetzlaff, J.; Mulrow, C.; Gøtzsche, P.C.; Ioannidis, J.P.A.; Clarke, M.; Devereaux, P.J.; Kleijnen, J.; Moher, D. The PRISMA Statement for Reporting Systematic Reviews and Meta-Analyses of Studies That Evaluate Health Care Interventions: Explanation and Elaboration. J. Clin. Epidemiol. 2009, 62, e1-e34. [CrossRef] 
30. Armijo-Olivo, S.; Stiles, C.R.; Hagen, N.A.; Biondo, P.D.; Cummings, G.G. Assessment of Study Quality for Systematic Reviews: A Comparison of the Cochrane Collaboration Risk of Bias Tool and the Effective Public Health Practice Project Quality Assessment Tool: Methodological Research. J. Eval. Clin. Pract. 2012, 18, 12-18. [CrossRef] [PubMed]

31. Shea, B.J.; Reeves, B.C.; Wells, G.; Thuku, M.; Hamel, C.; Moran, J.; Moher, D.; Tugwell, P.; Welch, V.; Kristjansson, E.; et al. AMSTAR 2: A Critical Appraisal Tool for Systematic Reviews That Include Randomised or Non-Randomised Studies of Healthcare Interventions, or Both. BMJ 2017, 358. [CrossRef]

32. OCEBM Levels of Evidence Working Group. The Oxford 2011 Levels of Evidence. Oxford Centre for Evidence-Based Medicine. 2011. Available online: http:/ / www.cebm.net/index.aspx?o=5653 (accessed on 13 May 2021).

33. Nappi, R.E.; Benedetto, C.; Campolo, F.; Martella, S.; Tosti, C.; Cianci, A.; Caruso, S.; Guaschino, S.; Grimaldi, E.; Bagolan, M.; et al. Efficacy, Tolerability and Safety of a New Medical Device, Monurelle Biogel ${ }^{\circledR}$ Vaginal Gel, in the Treatment of Vaginal Dryness: A Randomized Clinical Trial in Women of Reproductive Age. Eur. J. Obstet. Gynecol. Reprod. Biol. 2016, 203, 82-88. [CrossRef]

34. Schiavi, M.C.; Di Tucci, C.; Colagiovanni, V.; Faiano, P.; Giannini, A.; D’Oria, O.; Prata, G.; Perniola, G.; Monti, M.; Zullo, M.A.; et al. A Medical Device Containing Purified Bovine Colostrum (Monurelle Biogel) in the Treatment of Vulvovaginal Atrophy in Postmenopausal Women: Retrospective Analysis of Urinary Symptoms, Sexual Function, and Quality of Life. Low Urin. Tract Symptoms 2019, 11, O11-O15. [CrossRef] [PubMed]

35. Stefani, C.; Liverani, C.A.; Bianco, V.; Penna, C.; Guarnieri, T.; Comparetto, C.; Monti, E.; Valente, I.; Pieralli, A.L.; Fiaschi, C.; et al. Spontaneous Regression of Low-Grade Cervical Intraepithelial Lesions Is Positively Improved by Topical Bovine Colostrum Preparations (GINEDIE ${ }^{\circledR}$ ). A Multicentre, Observational, Italian Pilot Study. Eur. Rev. Med. Pharmacol. Sci. 2014, 18, 728-733.

36. Jones, A.W.; Cameron, S.J.S.; Thatcher, R.; Beecroft, M.S.; Mur, L.A.J.; Davison, G. Effects of Bovine Colostrum Supplementation on Upper Respiratory Illness in Active Males. Brain. Behav. Immun. 2014, 39, 194-203. [CrossRef] [PubMed]

37. Jones, A.W.; Thatcher, R.; March, D.S.; Davison, G. Influence of 4 Weeks of Bovine Colostrum Supplementation on Neutrophil and Mucosal Immune Responses to Prolonged Cycling. Scand. J. Med. Sci. Sports 2015, 25, 788-796. [CrossRef]

38. Jones, A.W.; March, D.S.; Thatcher, R.; Diment, B.; Walsh, N.P.; Davison, G. The Effects of Bovine Colostrum Supplementation on in Vivo Immunity Following Prolonged Exercise: A Randomised Controlled Trial. Eur. J. Nutr. 2019, 58, 335-344. [CrossRef]

39. Davison, G.; Jones, A.W.; Marchbank, T.; Playford, R.J. Oral Bovine Colostrum Supplementation Does Not Increase Circulating Insulin-like Growth Factor-1 Concentration in Healthy Adults: Results from Short- and Long-Term Administration Studies. Eur. J. Nutr. 2020, 59, 1473-1479. [CrossRef]

40. Hałasa, M.; Maciejewska, D.; Baśkiewicz-Hałasa, M.; Machaliński, B.; Safranow, K.; Stachowska, E. Oral Supplementation with Bovine Colostrum Decreases Intestinal Permeability and Stool Concentrations of Zonulin in Athletes. Nutrients 2017, 9, 370. [CrossRef] [PubMed]

41. Kotsis, Y.; Mikellidi, A.; Aresti, C.; Persia, E.; Sotiropoulos, A.; Panagiotakos, D.B.; Antonopoulou, S.; Nomikos, T. A LowDose, 6-Week Bovine Colostrum Supplementation Maintains Performance and Attenuates Inflammatory Indices Following a Loughborough Intermittent Shuttle Test in Soccer Players. Eur. J. Nutr. 2018, 57, 1181-1195. [CrossRef]

42. Kotsis, Y.; Methenitis, S.; Mikellidi, A.; Aresti, C.; Persia, E.; Antonopoulou, S.; Nomikos, T. Changes of Rate of Torque Development in Soccer Players after a Loughborough Intermittent Shuttle Test: Effect of Bovine Colostrum Supplementation. Isokinet. Exerc. Sci. 2020, 28, 59-72. [CrossRef]

43. March, D.S.; Jones, A.W.; Thatcher, R.; Davison, G. The Effect of Bovine Colostrum Supplementation on Intestinal Injury and Circulating Intestinal Bacterial DNA Following Exercise in the Heat. Eur. J. Nutr. 2019, 58, 1441-1451. [CrossRef] [PubMed]

44. Morrison, S.A.; Cheung, S.S.; Hurst, R.D.; Cotter, J.D. Cognitive Function and Blood-Brain Barrier Permeability during Exercise in the Heat: Effect of Fitness and Bovine Colostrum Supplementation. J. Therm. Biol. 2013, 38, 374-383. [CrossRef]

45. Morrison, S.A.; Cheung, S.S.; Cotter, J.D. Bovine Colostrum, Training Status, and Gastrointestinal Permeability during Exercise in the Heat: A Placebo-Controlled Double-Blind Study. Appl. Physiol. Nutr. Metab. 2014, 39, 1070-1082. [CrossRef] [PubMed]

46. Shing, C.M.; Peake, J.M.; Suzuki, K.; Jenkins, D.G.; Coombes, J.S. A Pilot Study: Bovine Colostrum Supplementation and Hormonal and Autonomic Responses to Competitive Cycling. J. Sports Med. Phys. Fit. 2013, 53, 490-501.

47. Aunsholt, L.; Jeppesen, P.B.; Lund, P.; Sangild, P.T.; Ifaoui, I.B.R.; Qvist, N.; Husby, S. Bovine Colostrum to Children with Short Bowel Syndrome: A Randomized, Double-Blind, Crossover Pilot Study. JPEN J. Parenter. Enteral. Nutr. 2014, 38, 99-106. [CrossRef]

48. Balachandran, B.; Dutta, S.; Singh, R.; Prasad, R.; Kumar, P. Bovine Colostrum in Prevention of Necrotizing Enterocolitis and Sepsis in Very Low Birth Weight Neonates: A Randomized, Double-Blind, Placebo-Controlled Pilot Trial. J. Trop. Pediatr. 2017, 63, 10-17. [CrossRef]

49. Barakat, S.H.; Meheissen, M.A.; Omar, O.M.; Elbana, D.A. Bovine Colostrum in the Treatment of Acute Diarrhea in Children: A Double-Blinded Randomized Controlled Trial. J. Trop. Pediatr. 2020, 66, 46-55. [CrossRef]

50. Juhl, S.M.; Ye, X.; Zhou, P.; Li, Y.; Iyore, E.O.; Zhang, L.; Jiang, P.; van Goudoever, J.B.; Greisen, G.; Sangild, P.T. Bovine Colostrum for Preterm Infants in the First Days of Life: A Randomized Controlled Pilot Trial. J. Pediatr. Gastroenterol. Nutr. 2018, 66, 471-478. [CrossRef]

51. Patıroğlu, T.; Kondolot, M. The Effect of Bovine Colostrum on Viral Upper Respiratory Tract Infections in Children with Immunoglobulin A Deficiency. Clin. Respir. J. 2013, 7, 21-26. [CrossRef] 
52. Rathe, M.; De Pietri, S.; Wehner, P.S.; Frandsen, T.L.; Grell, K.; Schmiegelow, K.; Sangild, P.T.; Husby, S.; Müller, K. Bovine Colostrum Against Chemotherapy-Induced Gastrointestinal Toxicity in Children with Acute Lymphoblastic Leukemia: A Randomized, Double-Blind, Placebo-Controlled Trial. JPEN J. Parenter. Enteral. Nutr. 2020, 44, 337-347. [CrossRef]

53. Saad, K.; Abo-Elela, M.G.M.; El-Baseer, K.A.A.; Ahmed, A.E.; Ahmad, F.-A.; Tawfeek, M.S.K.; El-Houfey, A.A.; Aboul Khair, M.D.; Abdel-Salam, A.M.; Abo-Elgheit, A.; et al. Effects of Bovine Colostrum on Recurrent Respiratory Tract Infections and Diarrhea in Children. Medicine 2016, 95, e4560. [CrossRef]

54. Duff, W.R.D.; Chilibeck, P.D.; Rooke, J.J.; Kaviani, M.; Krentz, J.R.; Haines, D.M. The Effect of Bovine Colostrum Supplementation in Older Adults during Resistance Training. Int. J. Sport Nutr. Exerc. Metab. 2014, 24, 276-285. [CrossRef] [PubMed]

55. Eslamian, G.; Ardehali, S.H.; Baghestani, A.-R.; Vahdat Shariatpanahi, Z. Effects of Early Enteral Bovine Colostrum Supplementation on Intestinal Permeability in Critically Ill Patients: A Randomized, Double-Blind, Placebo-Controlled Study. Nutrition 2019, 60, 106-111. [CrossRef]

56. Caysido, E.C.; Caysido, E.C. Effect of bovine colostrum on the absolute neutrophil count of pediatric patients with acute lymphocytic leukemia undergoing chemotherapy-A double blind randomized placebo controlled study. In Proceedings of the 2nd World Congress on Pediatrics and Clinical Pediatrics, Edinburgh, Scotland, 12-13 June 2019.

57. Barakat, S.H.M.; Omar, O.; Meheissen, M. Bovine Colostrum in the Treatment of Acute Diarrhea in Egyptian Children: A Randomised Double-Blinded, Placebo-Controlled Trial. Pediatrics 2019, 144, 235. [CrossRef]

58. Donowitz, J.; Alam, M.; Kabir, M.; Ferdous, T.; Zerin, A.; Nayak, U.; Haque, R.; Petri, W.A. PTM202, A Bovine Colostrum Based Nutritional Supplement, Decreases the Enteric Inflammation of Environmental Enteric Dysfunciton in Bangladeshi Infants. Am. J. Trop. Med. Hygiene 2019, 101, 194.

59. Oloroso-Chavez, K.; Andaya, P.; Wong, C. OR082 Bovine Colostrum Supplementation in Respiratory Allergies According to Sensitization: Subgroup Analysis of Randomized Controlled Trial. Ann. Allergy Asthma Immunol. 2017, 119, S11-S12. [CrossRef]

60. March, D.S.; Marchbank, T.; Playford, R.J.; Jones, A.W.; Thatcher, R.; Davison, G. Intestinal Fatty Acid-Binding Protein and Gut Permeability Responses to Exercise. Eur. J. Appl. Physiol. 2017, 117, 931-941. [CrossRef]

61. Martin, G.R.; Wallace, L.E.; Hartmann, B.; Holst, J.J.; Demchyshyn, L.; Toney, K.; Sigalet, D.L. Nutrient-Stimulated GLP-2 Release and Crypt Cell Proliferation in Experimental Short Bowel Syndrome. Am. J. Physiol. Gastrointest. Liver Physiol. 2005, 288, G431-G438. [CrossRef] [PubMed]

62. Blair, M.; Kellow, N.J.; Dordevic, A.L.; Evans, S.; Caissutti, J.; McCaffrey, T.A. Health Benefits of Whey or Colostrum Supplementation in Adults $\geq 35$ Years; a Systematic Review. Nutrients 2020, 12, 299. [CrossRef] 